\title{
Towards a Neo-Copenhagen Interpretation of Quantum Mechanics
}

\author{
Willem M. de Muynck \\ Theoretical Physics, Eindhoven University of Technology, \\ POB 513, 5600 MB Eindhoven, The Netherlands \\ E-mail: W.M.d.Muynck@tue.nl
}

\begin{abstract}
The Copenhagen interpretation is critically considered. A number of ambiguities, inconsistencies and confusions are discussed. It is argued that it is possible to purge the interpretation so as to obtain a consistent and reasonable way to interpret the mathematical formalism of quantum mechanics, which is in agreement with the way this theory is dealt with in experimental practice. In particular, the essential role attributed by the Copenhagen interpretation to measurement is acknowledged. For this reason it is proposed to refer to it as a neo-Copenhagen interpretation.
\end{abstract}

\section{Introduction}

Does quantum mechanics describe 'just the phenomena', or does it describe 'reality behind the phenomena'? Fifty or sixty years ago, under the influence of logical positivism/empiricism the majority of physicists would presumably have given a positive answer to the first question. Even today the influence of empiricism on the interpretation of the mathematical formalism of quantum mechanics is felt when using the term 'observable' to refer to a physical quantity represented by a Hermitian operator. And even though neither Bohr nor Heisenberg can be reckoned a logical positivist, their Copenhagen interpretation has been couched in the empiricist language of 'phenomena'. In particular, the emphasis placed on the essential role played by the measurement arrangement has fostered the idea that quantum mechanics is just dealing with phenomena to be observed in the course of a measurement.

But, in the meantime there has been a remarkable change of attitude. Following Einstein's criticism of the Copenhagen interpretation, to the effect that "Physics 
is an attempt conceptually to grasp reality as it is thought independent of its being observed" ([1], p. 81), there has been a tendency to try to interpret quantum mechanics as a description of an objective microscopic reality, 'objective' to be understood in the sense of 'being independent of the observer including his measuring instruments'. For instance, Bell [2]: "However the idea that quantum mechanics, our most fundamental physical theory, is exclusively even about the results of experiments would remain disappointing," and: "To restrict quantum mechanics to be exclusively about piddling laboratory operations is to betray the great enterprise." Indeed, in quantum mechanics textbooks of the last few decades hardly any reference is being made at all to the measurement arrangement. Quantum mechanics is presented as describing microscopic reality as it is supposed to be independently of any measurement. Interpretations like the many-worlds interpretation (Everett [3]) have been especially devised so as to expel measurement from the formulation of the theory. In the following I will refer to such interpretations as 'objectivisticrealist interpretations of (the mathematical formalism of) quantum mechanics' (cf. section 11). It is the purpose of the present paper to question the appropriateness of such objectivistic-realist interpretations, and to develop an interpretation in which the Copenhagen concern about the relation established by measurement between the microscopic object and the macroscopic world, is duly taken into account.

Unfortunately, the Copenhagen interpretation is far from unambiguous. Thus, Bohr's view with respect to the state vector is an instrumentalist one, in which the state vector is considered as 'just an instrument for calculating measurement results', thus opposing the realist view of an electron as a wave packet flying around in space. By contrast, even though Bohr usually remained at a purely conceptual level, and refrained from making ontological assertions, with respect to quantum mechanical observables ("physical quantities") his interpretation appears to be a realist one (Folse (4]), be it of a contextualistic blend in which a physical quantity is well-defined only within the context of the measurement arrangement serving to measure that quantity. In this respect it should be also noted that both Bohr and Heisenberg virtually equate a 'measurement result' (i.e. a value of a quantum mechanical observable) with a property of the microscopic object, possessed by the object during (Bohr) or after (Heisenberg) the measurement. By von Neumann's projection postulate this contextualistic-realist interpretation of observables is extended to quantum mechanical states.

In the following sections I will first give a critical discussion of a number of features of the Copenhagen interpretation. It will be seen that Feyerabend's [5] judgment that the 'Copenhagen point of view' is "not a single idea but a mixed bag of interesting conjectures, dogmatic declarations, and philosophical absurdi- 
ties" is completely justified. Moreover, a number of inconsistencies will be found. Nevertheless, the tendency to dismiss as irrelevant the Copenhagen concern with measurement is certainly unwarranted, and -in disagreement with the often-heard assertion that the best explanation of the quantum phenomena is that reality is objectively described by quantum mechanics- not even plausible. On the contrary, the fact that all knowledge we have about the quantum world is obtained by means of measurement endorses Bohr's intuition that our view of the world, laid down in our theories, must be colored by the interaction between object and measuring instrument. Therefore it is necessary to take this element of the Copenhagen interpretation very seriously. It is important to note already here, however, that this does not imply that it is possible to maintain a contextualistic-realist interpretation in the Copenhagen sense referred to above. Instead, an empiricist interpretation is proposed, in which a quantum mechanical observable refers to the measuring instrument rather than to the microscopic object (cf. section 11).

In order to avoid misunderstandings it should be stressed here that in the following a 'measuring instrument' is always taken to be a material object the microscopic object is allowed to physically interact with. The human observer and his consciousness can be left out of consideration. They are assumed not to have any physical influence on the measuring process after the measurement arrangement has been set up. Actually, in a quantum measurement the relation of a human observer to a measuring instrument is not different from that in classical physics: his looking at the (macroscopic) pointer of a quantum mechanical measuring instrument will influence the measurement result (the pointer position) just as little as it does in

a classical measurement. Nowadays, quantum measurements are often completely automated. The role of the human observer may even be restricted to looking at the graphs produced by his printer. Therefore in the following all allusion to 'mind', 'consciousness', 'free will', and 'psychophysical parallelism' will be ignored as being irrelevant to the subject. This implies that certain issues, like von Neumann's 'infinite regress', 'Wigner's friend', the 'many-minds interpretation', and 'subjectivity' will not be discussed.

\section{Completeness of quantum mechanics}

\subsection{Two notions of completeness}

In the discussion between Einstein and Bohr on 'completeness of quantum mechanics' it is necessary to distinguish two different notions of 'completeness', to be re- 
ferred to as 'completeness in a wider sense' and 'completeness in a restricted sense', respectively. The first notion is related to the impossibility of subquantum theories, and can be formulated as follows:

No subquantum theory can yield a more detailed description of physical reality than is provided by quantum mechanics (completeness in a wider sense).

A reason to believe in 'completeness in a wider sense' might be a positivist fear of the metaphysical, rejecting hidden variables because of their unobservable, and hence metaphysical, character. As observed by Feyerabend [5], Bohr accepted von Neumann's "proof" of the impossibility of hidden variables completing the quantum formalism ([6], section IV.2), currently known to lack cogency (Bell [7]). Yet, this was not the issue for Einstein when challenging Bohr's 'completeness' idea. The issue was rather different, viz.

The quantum mechanical description of a particle cannot be completed by determining precise values of position $\mathbf{r}$ and momentum $\mathbf{p}$, because the disturbing influence of the measuring instrument does not allow such a determination (completeness in a restricted sense).

The reason why Bohr thought quantum mechanics to be a complete theory was not any fear of the metaphysical. Instead, it is the existence of the 'quantum of action', represented by Planck's constant $h$, which is responsible for the impossibility of a simultaneous precise determination of position and momentum of a particle. For Bohr the concomitant impossibility of having a vanishing interaction between microscopic object and measuring instrument implies the impossibility of a sharp distinction between these objects, which therefore constitute an indivisible whole, manifesting itself as a 'quantum phenomenon' (this is known as Bohr's quantum postulate, e.g. [8]). Due to this circumstance position and momentum are each defined with a certain latitude, which latitudes satisfy the Heisenberg uncertainty relation. This issue is characteristic of quantum mechanics itself, and is quite independent of the question of hidden variables (which transcends the domain of quantum mechanics). It is 'completeness in a restricted sense' that is the issue in the discussion between Bohr and Einstein on the so-called 'thought experiments', culminating in the Einstein-Podolsky-Rosen (EPR) proposal [9], which should be seen as an ultimate attempt from Einstein's part to prevent an appeal by Bohr to the measurement interaction in countering his objections.

Viewed in this way, the discussion between Einstein and Bohr on the completeness of quantum mechanics is not at all about whether quantum mechanics is a 'theory of everything', not to be surpassed by any other more detailed theory. It is rather about the interpretation of quantum mechanics, viz. whether a realist 
interpretation of quantum mechanical observables can be either objectivistic (Einstein) or must be contextualistic (Bohr). It was Einstein's conviction that a sound physical theory must yield a description of an objective reality, and not merely of a reality that is in interaction with an observer, or even with a measuring instrument. The moon is there when nobody looks. The properties of matter, like electric conductivity, radioactivity, etc., do not seem to be dependent on our observations; we should try to devise theories describing these properties as being independent of any observation. This idea is at the basis of the notion of 'element of physical reality', introduced in the EPR paper 9], corresponding to a physical quantity the value of which can be predicted with certainty without in any way disturbing the system (cf. section [5). By Bohr [10] the unambiguity of this notion was challenged precisely because of its non-contextuality, ignoring that the (whole) experimental arrangement must be taken into account when discussing quantum phenomena.

It is important to stress here that by EPR an element of physical reality was presented as a quantum mechanical measurement result, which, for this reason, could be equated by Bohr to a 'quantum phenomenon'. Indeed, the discussion took place completely within the domain of quantum mechanics. No hidden variables, hence, no 'completeness in a wider sense' were involved. As far as hidden variables might be thought to be involved, the question just is whether the quantum mechanical observables themselves can play the roles of hidden variables, in the sense that to a particle a well-defined value of an observable can be attributed, possessed prior to, and independently of measurement. However, this is a matter of the interpretation of quantum mechanics (either objectivistic-realist or contextualistic-realist) rather than a fundamental change to a more encompassing theory. It should be borne in mind that this focussing of the attention on quantum mechanical notions has severely restricted the scope of physical reasoning. Thus, in this way the possibility is not taken into account that Bohr might be right in attributing a contextual meaning to quantum mechanics, but that Einstein's 'element of physical reality' might be given an unambiguous meaning when viewed as a non-quantum mechanical concept. By disregarding the latter possibility it became possible that Bohr's victory with respect to the issue of 'completeness in a restricted sense' was generally misinterpreted as a victory with respect to 'completeness in a wider sense', denouncing Einstein's introduction of 'elements of physical reality' as a metaphysical endeavor.

The idea that quantum mechanics might be 'complete in a wider sense' is a very unusual one, not applicable to any of the physical theories developed in the past. Therefore it is not clear at all why we might want to apply it to quantum mechanics. Admittedly, this theory has a very large domain of application, and it is not clear under which physical circumstances its boundaries might come into sight. But this 
was true as well for the theory of classical mechanics, including Maxwell's field theory, which encompasses virtually all of physics known 100 years ago (compare Kelvin [11]). Nevertheless, $20^{\text {th }}$ century physics has been dominated by quantum mechanics and relativity theory, and the classical theory has been recognized to be valid only if masses are sufficiently large and velocities are small compared to the velocity of light. Quantum mechanics does not seem to be so well understood that something analogous could not be expected (for instance, for measurement processes monitoring very short times, cf. section 12).

The idea that quantum mechanics is 'complete in a wider sense' can hardly be attributed to either Einstein, Bohr or Heisenberg, who were open-minded to the possibility that quantum mechanics may have to be superseded by still more advanced theories. If the idea of 'completeness in a wider sense' can be attributed to the Copenhagen interpretation at all, it is because this interpretation is not a consistent theory, but a set of ideas stemming from different sources. One such source is the empiricist philosophy of science which was dominating the first half of the $20^{\text {th }}$ century. In a certain fundamentalist form (often referred to as anti-realism) the empiricist philosophy not only advocated to be very cautious about theoretical concepts not based on observation, but it even declared objects corresponding to such concepts to be non-existent (for instance, atoms, the world aether). If taken seriously, application of this advice to atoms would presumably even have been an impediment to their experimental discovery. Although it may not be advisable to assume the physical existence of every theoretical notion we may be able to think of, the opposite attitude does seem to be equally unproductive. As a means of promoting science, 'fear of the metaphysical' has its boundaries. Sometimes a leap of imagination may be advantageous.

Up to now our experience has been that behind the phenomena described by a certain theory there is a wealth of new physics to be described by more penetrating theories. Thus, the behavior of a billiard ball as observed by a billiard player is adequately described by the classical theory of rigid bodies. However, we need solid state physics to take into account its atomic constitution. By analogy, it would be rather frivolous to assume that quantum mechanics is the "theory of everything", never to be superseded by more encompassing (subquantum) theories. Even though at this moment we do not have any experimental indication with respect to the boundaries of the domain of application of quantum mechanics (at least in its generalized form, cf. section 10), the idea of 'completeness in a wider sense' would be inappropriate from a methodological point of view. As a matter of fact, it is by now well known that the standard formalism of quantum mechanics, as presented in quantum mechanics textbooks, is not able to describe all measurements possible 
within the quantum mechanical domain (e.g. de Muynck [12]). For this reason, at least textbook quantum mechanics cannot be 'complete in a wider sense'. It will not be demanded in developing a neo-Copenhagen interpretation of quantum mechanics.

\subsection{Objectivity and contextuality}

There is yet another lesson to be learned from the billiard ball analogy. It is widely assumed that, in contrast to quantum mechanics, classical mechanics yields an $o b$ jective description of reality, not needing any reference to observation. From the example we see that this is not generally true. Rigidity is not an objective property of a billiard ball. Due to its atomic constitution, a billiard ball behaves as a rigid body only under certain circumstances. If hit hard enough it may start to vibrate, and may even split. Hence, the theory of rigid bodies is applicable only within a certain domain of experimentation, and, therefore, has only a contextual meaning, quite analogous to Bohr's view on the contextual meaning of quantum mechanical observables. As far as Einstein's idea of the desirability of an objective description might have its origin in a classical paradigm, this seems to be unwarranted. Moreover, it is evident that, analogous to Bohr's contention with respect to quantum mechanical observables, for a rigid body description of a billiard ball the context is determined by the whole experimental arrangement. This implies that 'rigidity of a billiard ball' is a contextual property even if no observation is made at all. Einstein's requirement of objectivity of the quantum mechanical description might be justified if quantum mechanics were 'complete in a wider sense'. This seems to be too strong a requirement, though.

However, Einstein's requirement of 'objectivity' is not related to the issue of 'completeness in a wider sense'. Indeed, his quarrel with Bohr was about 'completeness in a restricted sense'. It was unacceptable to Einstein that properties of a world, existing independently of the observer, would depend on its being observed. Indeed, it would be preposterous to assume that the rigidity of a billiard ball is dependent on its being looked at, or that the moon would not exist if it is not observed by any observer. The billiard ball example can teach us how this conundrum can be solved, both for classical and quantum mechanics. For this purpose it is advantageous to assume 'incompleteness in a wider sense' of these theories.

It is important to take into account the physical reason why a billiard ball is rigid, even if it consists of vibrating atoms. This reason, of course, is that, due to the tight bindings between the atoms within the ball, the vibrations are so small that they are not observed at the macroscopic level of observation to which the theory 
of rigid bodies is applicable. We should distinguish 'reality' from our 'description of reality'. A billiard ball is not "really" a rigid body; we can only describe it as one as long as it behaves accordingly. Hence, as far as rigidity is a property of the ball, it is a contextual property. Taking into account atomic vibrations makes this clear. Moreover, it demonstrates that for a description of these vibrations we need a theory different from rigid body theory (for instance, an atomic theory of the solid state). The contextual meaning of rigid body theory is evident because the concept of 'rigidity' loses its meaning when the atomic vibrations are no longer negligible. Actually, we should distinguish two concepts of 'rigidity', the 'rigidity' concept of rigid body theory being quite different from that of an atomic theory of the solid state. An interesting question is also whether, in contrast to the concept of 'rigidity' of rigid body theory, the atomic theory does yield an objective description of atomic vibrations, or whether under certain conditions these vibrations may lose their meaning too. However, this subject will not be pursued here any further.

For quantum mechanics the situation may be analogous. Submicroscopic (hidden variables) concepts of position and momentum may be different from the corresponding quantum mechanical concepts. Bohr may be right that, like rigidity, quantum mechanical observables too have a contextual meaning only. If so, a subquantum theory will be necessary to yield the objective description Einstein aspired to. Possibly, Einstein's requirement that quantum mechanics itself yield such a description is unnecessarily asking too much of this latter theory. We have some indications that this, indeed, may be the case. Thus, although at the time of the Bohr-Einstein discussion it was an open question whether values of quantum mechanical observables can be attributed to the microscopic object as objective properties (this possibility was denied by the Copenhagen interpretation on dubious grounds, cf. section [2.3), by the Kochen-Specker theorem and its generalizations [13, 14, 15, 16, 17, as well as by certain derivations of the Bell inequalities (e.g. [12], section 9.4.1), we are convinced now that such an attribution is impossible. Hence, in general it is impossible to assume that a free particle had a well-defined value of quantum mechanical momentum prior to measurement. This is one of the basic tenets of the Copenhagen interpretation, advocated in particular by Jordan [18, but generally felt to characterize this interpretation's view on the issue of (in)determinism, which (indeterministic) view -as is well known- was rejected by Einstein. His assertion that God does not play dice may be interpreted as expressing a conviction that in an ideal measurement an observation of a quantum mechanical measurement result can be explained because the observable had its value prior to the measurement. This is sometimes referred to as a principle of 'faithful measurement' (e.g. Redhead [19]). It seems that at least on the issue of the objectivity of quantum mechanical 
measurement results Bohr can be granted a victory over Einstein, even though it is questionable whether this extends to the issue of (in)determinism.

\subsection{To explain or not to explain}

The distinction between 'quantum mechanical measurement results' and 'subquantum elements of physical reality' will play an important role in my attempt to free the Copenhagen interpretation from a number of features reducing its trustworthiness. Jordan's 'creation-out-of-the-blue' philosophy is one of these. The Copenhagen abandonment of explanation of a measurement result by referring to a property of the microscopic object, possessed prior to measurement (for instance, according to Jordan [18] before a position measurement a particle is "neither here, neither there") has been a bone of contention to those believing, with Einstein, that a decent physical theory must tell us something about objective reality. Indeed, the Copenhagen idea of 'indeterminism' is based on a conviction that quantum mechanics cannot be completed by means of so-called hidden variables. This conviction is based on von Neumann's "proof" of the impossibility of the existence of hidden variables ( 6 , section IV.2), which, however, was demonstrated by Bell [7] not to be cogent (see also Feyerabend [20]). Hence, it is possible that here the Copenhagen interpretation underestimates the capacity of quantum mechanics to account for certain features of reality. Perhaps Heisenberg does not even follow Jordan all the way when he interprets his uncertainty relation as referring to mutual disturbance of position and momentum in a simultaneous measurement of these observables. Indeed, this seems to imply that an observable is not disturbed by an ideal measurement of that very observable, suggesting that, in agreement with the principle of 'faithful measurement', prior to measurement the observable must have had the same value it has after the measurement. Even though, due to the Kochen-Specker and Bell theorems, this cannot be literally true, Heisenberg's intuition might be correct in the sense that, contrary to Jordan's contention, a quantum mechanical measurement may yield evidence on a previously existing 'element of physical reality', which cannot be described by quantum mechanics, however, but which has to be described by a subquantum theory. Note, too, that Bohr has repeatedly warned against the idea that during measurement a quantum mechanical measurement result is created (as a property of the microscopic object).

Measurements of correlations between two observables may provide even more pressing arguments for the existence of 'subquantum elements of physical reality'. Consider, for instance, a free particle, for which the momentum observables $P(t)$ and $P\left(t^{\prime}\right)$ commute for all values of the times $t$ and $t^{\prime}$. It, therefore, follows from the 
mathematical formalism of quantum mechanics that two consecutive measurements of momentum of a free particle will yield the same measurement result. The perfect correlation between the results of two consecutive momentum measurements could be explained in a natural way by conservation of momentum, combined with the fact that, due to the commutativity of the momentum operators $P(t)$ and $P\left(t^{\prime}\right)$, the measurements of these observables do not mutually disturb. The perfect correlations of the measurement results would be completely unexplained if they were not reducible to some feature of the microscopic reality behind the measurement phenomena. A desire to explain a similar perfect correlation of quantum mechanical observables of two different particles in the EPR experiment may be responsible for the idea that, if the measured quantities cannot be objective properties of the particles, possessed prior to measurement, nonlocal influences between distant measurements must be involved.

Even though values of Hermitian operators (analogous to the rigidity concept of rigid body theory) cannot play the roles of 'elements of physical reality', this does not imply that they would not refer to some aspect of reality that is actually probed by the measurement of the corresponding observable. Thus, quantum mechanical momentum of a particle may refer to some subquantum notion of 'momentum', related to the quantum mechanical notion in a way analogous to the relation between the notion of 'rigidity' of rigid body theory and its representation in atomic solid state theory: conservation of momentum may be compared to conservation of the spherical shape of the surface of a billiard ball caused by the (approximate) preservation of the relative positions of the atoms; whereas under standard conditions these features strictly correspond to our observations, a description by the more detailed theory may account for deviations which become important under non-standard conditions.

Einstein's reluctance to grant completeness to quantum mechanics was not induced by any preference for the determinism of classical mechanics, but rather by the idea that our physical theories must tell us something about the world as it exists objectively and independently of measurement. Although this ideal may not be applicable to all physical theories (e.g. rigid body theory), and, in particular, not to quantum mechanics, it is not too far-fetched to assume with Bohr that quantum mechanics at least may tell something about a contextual reality that is in interaction with a measuring instrument. Nor does it mean that no other explanations can be attempted. As a matter of fact, within the Copenhagen interpretation such an attempt is made by invoking von Neumann's projection postulate, trying to explain the strict correlation between consecutively measured values of momentum by assuming that a momentum measurement projects the quantum state into an eigen- 
state of the measured observable. However, von Neumann's projection postulate is inapplicable to most measurement procedures (e.g. de Muynck [12, chapter 3). Moreover, as an explanation it does not seem to be very plausible if compared with the possibility that 'conservation of momentum' tells us something about a property of the object that is conserved while it is not interacting with a measuring instrument (an 'element of physical reality'). It seems evident that, if quantum mechanical observables have a contextual meaning only, such a property, if existing, must have a non-quantum mechanical nature.

Of course, it is possible to refrain from any explanation of correlations of consecutive quantum mechanical measurement results like the one discussed above, or of the strict (EPR) correlations obtained in a simultaneous measurement of the $z$ components of the spins of a spin- $1 / 2$ particle pair prepared in the singlet state. This would suit a strictly empiricist view of quantum mechanics (e.g. van Fraassen [21]), in which it is deemed sufficient that a physical theory just describes the phenomena, without any necessity to explain them. As is evident from its adoption of von Neumann's projection postulate, the Copenhagen interpretation does not hold to this strictly empiricist view. It does not refrain from explanation. On the contrary, it seems to be so inclined toward explanation that it is ready to accept a suspect procedure like von Neumann's projection postulate as a means of explaining correlations in consecutive or in joint measurements. It seems to me that, in devising a neoCopenhagen interpretation of quantum mechanics, we should maintain the demand that physical theories provide explanations, but we must accept that, like rigid body theory, also quantum mechanics may not explain everything. In particular, it does not explain why a certain measurement result is obtained when a measurement of a quantum mechanical observable is carried out. For such an explanation, if it exists, we will have to resort to more detailed (subquantum) theories, much in the same way rigidity of a billiard ball is explained by the tight bindings between the atoms within the ball, to be described by a sub-rigid body theory. If quantum mechanics is not 'complete in a wider sense', we may expect that subquantum theories will once be found. Far from being methodologically objectionable, subquantum (hidden variables) theories may be illuminating by allowing different levels of discourse.

\section{Individual-object (individual-particle) versus en- semble interpretation of the wave function}

The question of whether quantum mechanics is 'complete' is often formulated in terms of the wave function or state vector. Einstein was very clear at that. Accord- 
ing to him "the $\psi$-function is to be understood as the description not of a single system but of an ensemble of systems" (Einstein [22, p. 671). He thought that our inability to yield a more precise description than a statistical one is a consequence of our ignorance about the precise values of position and momentum of a particle. According to the Copenhagen interpretation such an ensemble interpretation is not suitable for quantum mechanics, because it is in disagreement with the idea of 'completeness in a restricted sense' (cf. section 2.11), disallowing an object to simultaneously have well-defined values of position and momentum ${ }^{1}$. According to the Copenhagen interpretation the wave function must be seen as a fundamentally probabilistic (as opposed to statistical) description of an individual object, allowing for the essential indeterminism which is a consequence of 'completeness in a restricted sense'. The difference between the Copenhagen individual-particle interpretation and Einstein's ensemble one marks their fundamentally different attitudes with respect to explanation of quantum mechanical measurement results.

Sometimes the distinction between an ensemble interpretation and an individualobject interpretation is characterized in terms of the distinction between an epistemic and an ontic interpretation, respectively (e.g. Primas [23]), juxtaposing 'description of our knowledge' to 'description of reality'. It seems to me, however, that this is a potentially misleading distinction, introduced in the first place to discredit an epistemic interpretation as being part of psychology, not physics. It should be noted, however, that, independently of its interpretation, a physical theory is a representation of our knowledge (about some part of reality), and, therefore, is always epistemic. This holds true for the wave function as well, independently of whether it is considered as a description of an individual particle or of an ensemble. Contrary to what is intimated by an ontic interpretation, an electron is not a wave function flying around in space. Electrons belong to physical reality, wave functions can be found in quantum mechanics textbooks.

I will not discuss here any further the question of whether the Copenhagen interpretation is ontic in the sense given above, because it seems to me that different adherents to this interpretation may give different answers (often couched in a terminology referring to the objectivism/subjectivism dichotomy). For instance, it seems evident that Bohr's (instrumentalist) interpretation of the wave function is not ontic. However, as already remarked in section 11 there has been a strong tendency towards an ontic interpretation, considering a wave function to describe the reality

\footnotetext{
${ }^{1}$ Here it is temporarily ignored that in the Copenhagen interpretation a quantum mechanical measurement result is generally interpreted as a post-measurement property of the object. The Copenhagen lack of distinction between pre- and post-measurement properties will be discussed in section 8
} 
of an individual microscopic object, much in the same way as the theory of rigid bodies seems to describe the reality of a billiard ball (see also section 11). Nowadays the 'epistemic' terminology has become acceptable again by stressing that the wave function must be seen as representing information which is available about the object. This would be a step forward if the experimental means, necessary to obtain this information, are not left out of consideration (which is seldom the case, however).

Unfortunately, reliance on the distinction 'epistemic versus ontic' has had a considerable impact. Thus, in the distinction between 'statistical' and 'probabilistic' interpretations of the quantum mechanical formalism it has been assumed that the latter interpretation implies indeterminism in an ontic sense rather than in the epistemic sense of lack of knowledge. It must be stressed, however, that we do not have any hard experimental evidence for such a contention. The arguments advanced by the Copenhagen and other interpretations in favor of an individual-particle interpretation of the wave function are based on an unwarranted lack of distinction between microscopic reality itself and its quantum mechanical description. For this reason I will not consider the epistemic/ontic dichotomy any further, but I will focus here on the individual-particle/ensemble dichotomy in which a quantum mechanical wave function may refer to either one of two different objects, both "ontically" existing in reality, viz. an individual object or an ensemble of such objects, realizing that in both cases the quantum mechanical description is an approximate one.

One prominent argument in favor of an ensemble interpretation of the wave function or state vector is the fact that quantum mechanical probabilities can be experimentally approached only by repeating an experiment a large number of times, and by determining relative frequencies of measurement outcomes. The possibility of an individual-particle interpretation then hinges on the question of whether all individual preparations in an ensemble are 'identical preparations', not allowing any specification by which they could be distinguished. By Von Neumann [6] this was taken up as signifying homogeneity of an ensemble described by a wave function (in contrast to the apparent inhomogeneity of a von Neumann ensemble described by a non-idempotent density operator). I will now discuss two reasons why I think that an individual-particle interpretation of the wave function is undesirable, even though at this moment this is not strictly falsifiable due to lack of any possibility to distinguish, other than by the measurement results obtained in quantum mechanical measurements, between individual realizations of members of an ensemble described by a wave function.

As a first reason, let us consider a particle impinging on a double-slit system. 
Nowadays such experiments are no longer 'thought experiments'. For instance, in neutron interferometry such experiments are routinely performed (e.g. [24, 25, 26]). In an individual-particle interpretation such experiments immediately entail a conceptual problem. As a matter of fact, the wave function is split into two parts, each corresponding to one of the slits. This is sometimes interpreted as 'the particle going through both slits at the same time'. A more appropriate interpretation might be thought to be the Copenhagen one, to the effect that within the experimental arrangement the particle concept is not well-defined, and a 'wave' terminology should be used to interpret what is going on (particle-wave duality). Unfortunately, the Copenhagen idea of particle-wave duality, asserting that, depending on the experimental arrangement, a quantum mechanical object "is" either a particle or a wave, must be considered obsolete by now. In interference experiments both particle and wave aspects can be observed within one and the same experimental arrangement. For instance, in interference experiments with electrons and neutrons it is possible to see a gradual development of the interference pattern, built up by local (particle-like) impacts exhibiting particle-like and wave-like behavior in one and the same measurement arrangement (compare figure 4.4 of de Muynck [12]). This experimentally demonstrates that in interference experiments the wave aspect of the phenomenon cannot be explained by considering an individual particle as a wave (which, incidentally, would be analogous to explaining rigidity of a billiard ball by means of a model of closely packed rigid atoms). For Bohr this has been occasion to revise his views, to the effect that electrons, protons and neutrons are considered by him to be always particles, whereas light would always be a wave, photons being artefacts of the quantum mechanical description (cf. Murdoch [27]).

Indeed, it is consistent with all experimental evidence to assume that electrons and neutrons maintain a particle-like behavior while passing through the interferometer. If detectors are placed within the interferometer, the particle is always found in one of the possible paths, never in both paths at the same time. Something similar is observed when a photon is allowed to impinge on a semi-transparant mirror (hence, it seems that in this respect Bohr's revised view is questionable as well). Whereas the wave function is split into approximately equal parts (transmitted and reflected parts), the photon travels one way or the other, as can be observed by putting photon counters in each of the outgoing directions. Evidently, if the wave function were interpreted as a description of a single particle or photon, the object (wave?) would be equally in both paths. This does not seem to be in agreement with the results of optical experiments, however.

In the Copenhagen probabilistic interpretation of the wave function a particle ontology is employed, but it is thought to be undefined whether the particle is in one 
path or the other; it might even jump to and fro between the paths. Analogously to the case of conservation of momentum, discussed above, we have no reason to believe that in a position measurement von Neumann projection is the reason that we find the object in one and the same beam when consecutive position measurements are carried out. For an explanation of this it is far more reasonable to appeal to inertia as a general property of matter, valid in the microscopic domain as well (although, possibly, not in the classical mechanical sense). The reason of the assumption of indeterminism by the Copenhagen interpretation might be also a consequence of an ill-founded idea of 'completeness of quantum mechanics in a wider sense', precluding a distinction between quantum mechanical measurement results and (non-quantum mechanical) properties of the microscopic object. Einstein's statistical (ensemble) interpretation of the wave function is no less in agreement with experiment, and its determinism in the sense of 'faithful measurement' seems to be quite a bit closer to what should be expected from a physical theory. For this reason it seems to be more promising to try to remedy shortcomings of this 'ensemble' approach than to keep trying to circumvent the paradoxes going with an individual-particle interpretation. One of these paradoxes is discussed in the next section. It yields a second reason to doubt the possibility of an individual-particle interpretation of the wave function, if the particle is considered in von Neumann's sense as a member of a homogeneous ensemble.

\section{4 (In)homogeneity of ensembles}

When the idea of 'homogeneity of an ensemble described by a pure state' is applied to an entangled state

$$
\left|\psi_{12}\right\rangle=\sum_{i j} c_{i j}\left|\alpha_{1 i}\right\rangle\left|\beta_{2 j}\right\rangle, c_{i j} \neq c_{1 i} c_{2 j}
$$

$\left\{\left|\alpha_{1 i}\right\rangle\right\}$ and $\left\{\left|\beta_{2 j}\right\rangle\right\}$ orthonormal sets in the Hilbert spaces of particles 1 and 2, respectively, we are confronted with a consistency problem. By taking a partial trace of the density operator $\rho_{12}=\left|\psi_{12}\right\rangle\left\langle\psi_{12}\right|$, the state of particle 1 is obtained as

$$
\rho_{1}=\operatorname{Tr}_{2} \rho_{12}=\sum_{j} r_{j}\left|\phi_{1 j}\right\rangle\left\langle\phi_{1 j}|,| \phi_{1 j}\right\rangle=\sum_{i} \frac{c_{i j}}{r_{j}^{1 / 2}}\left|\alpha_{1 i}\right\rangle, r_{j}=\| \sum_{i} c_{i j}\left|\alpha_{1 i}\right\rangle \|^{2} .
$$

The important point is that $\rho_{1}$ does not describe a pure state but a von Neumann ensemble, which, allegedly, is inhomogeneous. However, since particle 1 is a subsystem of the two-particle system described by the pure state (11), we do not have any means 
to distinguish between different members of the (allegedly homogeneous) ensemble, also if only one of the particles is considered. Hence, it would seem that the particle 1 ensemble must be homogeneous too. This poses the question of whether $\rho_{1}$, given by (22), describes a homogeneous or an inhomogeneous ensemble, the latter answer implying an inconsistency if the pure state (11) would correspond to a homogeneous one.

The question cannot be answered straightforwardly in an unambiguous way. As a matter of fact, for every density operator of the type (2) an entangled state of the form (11) can be constructed such that the density operator can be obtained by taking a partial trace. If pure states are homogeneous, this would seem to imply that ensembles of the type described by (21) must be homogeneous too. This is one way to try to prevent the above-mentioned inconsistency: consider all quantum mechanical ensembles, either pure states or mixtures, as homogeneous. This is actually done in the minimal interpretation advocated by e.g. Park and Band [28, 29]. In this way a problem of von Neumann's interpretation, caused by the non-uniqueness of the decomposition of the ensemble described by density operator (2) into distinct subensembles, might be solved. However, there is a different solution, viz., the one advocated by Einstein, considering all ensembles, both pure states as well as mixtures, as inhomogeneous. Hence, on the basis of the present considerations no definite answer can be given.

In order to discuss the problem of (in)homogeneity more fully, I will assume that in a homogeneous ensemble a quantum mechanical measurement produces a random sequence of measurement results which is homogeneous too. Here 'homogeneity of a random sequence' is taken in the sense of von Mises' theory of random sequences [30] according to the definition:

A random sequence is homogeneous if every allowed subsequence has the same relative frequency as the original sequence (homogeneity of a random sequence).

According to von Mises a subsequence is allowed if its members are selected using an algorithm that does not depend on the values of the selected members (for instance, in a random sequence consisting of 0 's and 1's, the criterion 'select all 0 's' is not allowed). Now the question of whether an ensemble, described by density operator $\rho=\sum_{j} r_{j}\left|\phi_{j}\right\rangle\left\langle\phi_{j}\right|$ is inhomogeneous, hinges on the question of whether there exists an allowed selection procedure to select subensembles described by state vectors $\left|\phi_{j}\right\rangle$ (which subensembles in general yield distinct measurement results if the state vectors are different for different $j$ ). As argued above, von Neumann's choice to answer this question affirmatively is less justified than it appear to be at first sight.

By d'Espagnat (31], chapter 7.2) a distinction has been drawn between proper 
and improper mixtures, described by the same density operator. In the case of a proper mixture, represented by density operator $\rho=\sum_{j} r_{j}\left|\phi_{j}\right\rangle\left\langle\phi_{j}\right|$, an allowed selection procedure exists, viz. selection on the basis of the parameter setting $j$ of the preparation apparatus corresponding to the preparation of the subensemble described by $\left|\phi_{j}\right\rangle$. This selection procedure is allowed because the parameter setting $j$ determines the state $\left|\phi_{j}\right\rangle$ rather than the other way around. According to the von Mises criterion a proper mixture must therefore be inhomogeneous.

When we apply the above reasoning to improper mixtures (obtained from a pure entangled state by partial tracing) the outcome is less unambiguous. The question is whether also in the case of an improper mixture an allowed selection procedure can be found yielding a subsequence with relative frequency differing from the one obtained from $\rho$. Using (11) and (2) (taking $\rho_{1}=\rho$ and $\phi_{1 j}=\phi_{j}$ ), a selection procedure could be contemplated on the basis of the existence of a polar decomposition (Schmidt [32]), to the effect that any two-particle state $\left|\psi_{12}\right\rangle$ can be written according to

$$
\left|\psi_{12}\right\rangle=\sum_{i} c_{i}\left|\alpha_{1 i}^{(s)}\right\rangle\left|\beta_{2 i}^{(s)}\right\rangle
$$

where $\left\{\left|\alpha_{1 i}^{(s)}\right\rangle\right\}$ and $\left\{\left|\beta_{2 i}^{(s)}\right\rangle\right\}$ are special orthonormal sets of eigenvectors of observables of particles 1 and 2, respectively, determined by the state vector $\left|\psi_{12}\right\rangle$ (these vectors turn out to be the eigenvectors of the reduced density operators $\rho_{1}$ and $\rho_{2}$, obtained from $\left|\psi_{12}\right\rangle\left\langle\psi_{12}\right|$ by partial tracing). Hence, for any (improper) mixture there exist two observables, $A_{1}^{(s)}$ and $B_{2}^{(s)}$ (having the vectors $\left|\alpha_{1 i}^{(s)}\right\rangle$ and $\left|\beta_{2 i}^{(s)}\right\rangle$ as eigenvectors, respectively) which are strictly correlated according to (3). Now a measurement of $B_{2}^{(s)}$ on particle 2 might seem to yield an allowed selection procedure for selecting subensembles of particle 1 described by the vectors $\left|\alpha_{1 i}^{(s)}\right\rangle$. Since the relative frequencies of a measurement performed on particle 1 will in general be different for different values of $i$, the ensemble corresponding to $\rho=\rho_{1}$ (in which the measurement results for particle 2 are ignored) would then be inhomogeneous. However, as will be seen next, there is a catch to this argument.

Another way to look at this problem is by not only considering a measurement of observable $B_{2}^{(s)}$ on particle 2 but also a simultaneously performed measurement of observable $A_{1}^{(s)}$ on particle 1, yielding two (correlated) sequences of measurement results. Due to the correlation, subsequences of measurement results of particle 1 can be selected, conditional on certain measurement results of particle 2. Once again, the question is whether this selection procedure is an allowed one. The answer to this question hinges on the (in)dependence of the value of observable $B_{2}^{(s)}$ (constituting the criterion of selection) with respect to the value of observable $A_{1}^{(s)}$. 
In general, measurements performed on two different particles can be considered to be independent. This suggests that the selection procedure is an allowed one. However, we do not have a general situation here. With respect to the measured observables state vector (3) is a very special one, warranting a strict correlation between the measurement results of the two particles. The selection procedure makes an essential use of this strict correlation, even to the effect that occasionally a measurement on one particle is interpreted as a measurement of the correlated observable of the other particle (compare the EPR experiment to be discussed in section (5). If this is taken into account, the selection procedure does not seem to be allowed any more, because in this experiment selection of a measurement result of particle 2 is equivalent to selection of the corresponding measurement result of particle 1, thus making the selection procedure depend on the value of the selected measurement result. On this basis homogeneity of improper mixtures could be thought to be maintainable.

However, this conclusion is changed if we take into account the possibility of measuring, in coincidence with $B_{2}^{(s)}$, an observable $A_{1}^{\prime}$ of particle 1 differing from $A_{1}^{(s)}$. In general, in the state (3) observables $A_{1}^{\prime}$ and $B_{2}^{(s)}$ are not strictly correlated, and their measurement results can be considered as independently obtained. This implies that, according to von Mises' definition, the ensemble represented by (2) can be considered as inhomogeneous. Homogeneity of mixtures (either proper or improper) seems to be maintainable only if the experimental possibility to split quantum mechanical ensembles into distinct subensembles is ignored. We will see in the following that this issue is closely related to the Copenhagen negligence of the difference between the notions of 'preparation' and 'measurement', causing a strong

focussing on measurement of the special observables $A_{1}^{(s)}$ and $B_{2}^{(s)}$ if the state is given by (3), and entailing a virtual absence of any discussion of measurement of other observables. This neglect has played a very confusing role in the discussions following the EPR proposal. Occasional references to a broader point of view, like the one by Hooker (33], section 5), to the effect that consideration of a joint measurement of position of particle 1 and momentum of particle 2 might provide an argument to be used in the EPR challenge of the Copenhagen interpretation, remained largely unnoticed.

\section{The Einstein-Podolsky-Rosen experiment}

The Einstein-Podolsky-Rosen experiment 9] can be considered as an ultimate attempt from Einstein's part to prove 'incompleteness of quantum mechanics', in the 
sense that quantum mechanics is not capable to account for the sharp values of both position and momentum that allegedly can be simultaneously attributed to a microscopic object. Earlier attempts had been defeated by Bohr by pointing to the disturbing influence of measurement, making such a simultaneous attribution impossible. Therefore the EPR proposal was devised so as to make it possible to obtain knowledge about a particle "without in any way disturbing the system." In the experiment a two-particle system is considered, described by an entangled state of the type (3), the two particles being prepared so as to be so far apart that a measurement on particle 1 does not influence particle 2. Due to the correlation between observables $A_{1}^{(s)}$ and $B_{2}^{(s)}$ expressed by (3) , it is possible according to EPR to infer the value of the quantum mechanical observable $B_{2}^{(s)}$ of particle 2 from the measurement result obtained by measuring observable $A_{1}^{(s)}$ on particle 1 . In this way information on particle 2 is obtained without in any way disturbing this particle.

A conclusion of 'incompleteness of quantum mechanics' is drawn by EPR for the special situation in which the expansion (3) of state vector $\left|\psi_{12}\right\rangle$ is not unique, in the sense that orthonormal sets $\left\{\left|\alpha_{1 i}^{\prime}\right\rangle\right\}$ and $\left\{\left|\beta_{2 i}^{\prime}\right\rangle\right\}$ of eigenvectors of observables $A_{1}^{\prime}$ and $B_{2}^{\prime}$, respectively, exist such that $\left|\psi_{12}\right\rangle$ can be also expressed according to

$$
\left|\psi_{12}\right\rangle=\sum_{i} c_{i}^{\prime}\left|\alpha_{1 i}^{\prime}\right\rangle\left|\beta_{2 i}^{\prime}\right\rangle
$$

The crucial point of the EPR reasoning is that observable $A_{1}^{\prime}$ is incompatible with $A_{1}^{(s)}$ (and analogously for observables $B_{2}^{(s)}$ and $B_{2}^{\prime}$ of particle 2). Because particle 2 cannot experience which of the two observables is measured on particle 1 , it is concluded by EPR that values of the incompatible observables $B_{2}^{(s)}$ and $B_{2}^{\prime}$ can be simultaneously attributed to particle 2. 'Incompleteness of quantum mechanics' follows from the circumstance that quantum mechanics is not able to describe a state of particle 2 in which both of these observables have sharp values.

It is important to note here that in reaching this conclusion EPR in an essential way make use of an objectivistic-realist interpretation of the mathematical formalism of quantum mechanics, since they assume quantum mechanical measurement results to correspond to objective properties of the microscopic object ('elements of physical reality'). It is precisely on this count that Bohr [10] challenged the EPR conclusion of incompleteness. According to Bohr's correspondence principle, to be discussed in section [6, quantum mechanical measurement results have a contextual meaning that is determined only by taking into account the 'whole experimental arrangement', including the measurement arrangement for particle 1. The actual presence of this arrangement is essential for obtaining knowledge on particle 2. According to Bohr, Einstein's definition of elements of physical reality of particle 2 is ambiguous because 
it does not take into account the experimental arrangement of particle 1. Bohr thought that if this is corrected the EPR challenge could be dealt with in precisely the same way earlier attempts by Einstein to prove incompleteness of quantum mechanics were countered.

However, Bohr's above-mentioned judgment is not completely reliable (e.g. Popper (34, p. 149), Jammer (35], p. 194), also Folse [4]). Admittedly, the issue of 'contextuality' is used by him also here, but not in the interactional sense involved in the 'quantum postulate'. Bohr's answer to EPR implies a change of interpretation, to the effect that for defining a physical quantity of particle 2 it is sufficient that a relation exist between particle 2 and the measuring instrument (which interacts only with particle 1). It is no longer deemed necessary that this relation (which was compared to a coordinate system by Bohr) be realized by an interaction, like it was before.

This change of interpretation from an interactional to a relational point of view has had a large impact on later developments, because it introduced into the interpretation of quantum mechanics an issue of 'nonlocality' which since that time has stayed with us. It was contended by Einstein that this nonlocality is just an artefact of Bohr's interpretation, combining contextuality of observables with an individual-particle interpretation of the state vector (i.e. completeness). According to him objectivity of observables could be restored by assuming an ensemble interpretation of the state vector (i.e. incompleteness): such an interpretation would allow to avoid the Copenhagen indeterminism (implying that a value of a quantum mechanical observable cannot be attributed to the object as a property possessed prior to measurement), and to interpret von Neumann projection, applied to particle 2 on the basis of a measurement on particle 1, as a selection of a subensemble. Unfortunately, in 1935 no Kochen-Specker or Bell theorems were available to put this into doubt. Nowadays we are convinced by these theorems that it is not possible to attribute values of all quantum mechanical observables as objective properties to the object, possessed independently of measurement, and that, therefore, Einstein's ensemble interpretation cannot be the final solution (e.g. Guy and Deltete [36]). This does not imply, however, that Bohr's nonlocal contextuality must be accepted. Its general acceptance is based on a number of confusions and inconsistencies which have entered the Copenhagen interpretation, to be discussed in the following sections. 


\section{Correspondence and complementarity}

According to Petersen [37], the correspondence principle is characteristic of the "Copenhagen spirit of quantum theory." The idea of 'correspondence' is an expression of the necessity felt to maintain as much as possible the use of classical concepts within the atomic domain. It is necessary to distinguish a weak and a strong form of the correspondence principle. In its weak form (e.g. Messiah [38, section 1.12) it is a requirement that the laws of microscopic physics must be formulated in such a way that in the limit of large quantum numbers (the 'classical limit') quantum mechanical results must agree with the results of classical mechanics. In its strong form the correspondence principle is valid not only in an asymptotic sense, but it is a requirement to be met by any measurement within the microscopic domain, independently of the values of the quantum numbers.

By the strong form of the correspondence principle the necessity is expressed of basing the description of the properties and manipulation of the measuring instruments on purely classical ideas, which are the ideas of macrophysics, thought to be the only means of unambiguous communication. According to Bohr a quantum phenomenon can be communicated only by using the classical terms by which the measurement arrangement is characterized. The measurement arrangement plays a key role in defining a quantum mechanical observable.

A quantum mechanical observable is exclusively defined by and within the context of the measurement serving to measure that observable; experimental arrangement and measurement results are to be described in classical terms (strong form of the correspondence principle).

Note that according to Bohr this does not mean that a quantum mechanical measurement process could not be described quantum mechanically. However, such a description would not be able to account for the macroscopic behavior of the measuring instruments, and, for this reason, would no longer allow to consider the process as a measurement process.

The idea of 'complementarity' is just an extension of the idea of 'correspondence'. If the definition of a quantum mechanical observable is restricted by its measurement arrangement, then mutual exclusiveness of measurement arrangements can explain why incompatible observables cannot simultaneously have sharp values. Thus,

Incompatible quantum mechanical observables correspond to mutually exclusive measurement arrangements, defining different aspects of reality which cannot be united in a single classical picture (complementarity). 
Bohr and Heisenberg applied the idea of 'complementarity' in the first place to position and momentum observables, which, due to complementarity, cannot be defined more accurately than is allowed by the Heisenberg uncertainty relations

$$
\Delta Q \Delta P \geq \hbar / 2,(\Delta Q)^{2}=\left\langle\Psi\left|(Q-\langle Q\rangle)^{2}\right| \Psi\right\rangle,(\Delta P)^{2}=\left\langle\Psi\left|(P-\langle P\rangle)^{2}\right| \Psi\right\rangle
$$

thus allegedly implementing the idea of 'completeness in a restricted sense' in the quantum mechanical formalism as a consequence of incompatibility of the corresponding operators: $[Q, P]_{-}=i \hbar$. By Heisenberg this was interpreted as a consequence of mutual disturbance in a simultaneous measurement of position and momentum, caused by mutual exclusiveness of the measurement arrangements serving to measure each of these observables separately in an ideal way.

It should be noticed that the interpretations of Bohr's and Heisenberg's are rather different from each other. By Heisenberg the quantities $\Delta Q$ and $\Delta P$ are interpreted as measures of the measurement accuracies of simultaneously performed measurements of position and momentum, respectively. Heisenberg ([39], section II.2) explicitly noted that inequality (44) must be seen as a property of the microscopic object, valid in its post-measurement state, and, therefore, to be seen as an objective property of the microscopic object after it has ceased to interact with the measuring instrument. For Bohr the inequality marks a limitation of our possibility to apply to a microscopic object the classical notions of position and momentum (latitudes of definition) during a simultaneous measurement of these quantities. Of course, if every initial state were prepared by a simultaneous measurement of position and momentum, the difference between Bohr and Heisenberg would be inconsequential. However, although all measurements are also preparations, the converse is not very plausible. Equating arbitrary preparations realized in nature with measurements would stretch the definition of 'measurement' rather too much. The seeming agreement on the meaning attributed by Bohr and Heisenberg to the Heisenberg uncertainty relations, marks the fundamental failure of the Copenhagen interpretation to distinguish 'preparation' and 'measurement' (cf. section 8). It was realized only a very long time after the inception of the idea of 'complementarity' (Ballentine [40]) that it is inconsistent to interpret (40)-which is a property of the initial (pre-measurement) state $|\Psi\rangle$ - as referring to a subsequent measurement.

Although presumably not completely justified, the correspondence principle has often been considered as demonstrating an inclination of the Copenhagen interpretation towards positivism/empiricism. Indeed, the insight that an account of a quantum mechanical measurement must be based on a so-called 'observation language', or, at least, on an independently tested theory (viz. classical mechanics), 
was consistent with the logical positivist/empiricist ideas. We should be cautious with such a conclusion, however. Empiricism does not seem to be involved in Bohr's characterization of the quantum phenomenon by 'the experiment as a whole', supposed to refer to observations obtained under specific circumstances including an account of the whole experimental arrangement. Nor does the Copenhagen usage of interpreting measurement results as (classical) properties of the microscopic object rather than as pointer readings of a (macroscopic) measuring instrument point into the direction of empiricism. Above all, the non-vanishing value of the "quantum of action' is the characterizing feature stressed by Bohr, making it impossible to draw a sharp distinction between microscopic object and measuring instrument. Heisenberg (41], p. 145), while distinguishing between the reality of the positivistic sense impressions of an observer and the reality of objects and events dealt with in atomic physics, even explicitly denies that the Copenhagen interpretation would be a positivistic one (see also section 11).

Nowadays the reliance of the correspondence principle on a classical description of measurement is recognized as a severe drawback. It has been realized that it is impossible to describe information transfer from a microscopic object to a measuring instrument by means of classical mechanics alone. Admittedly, a measuring instrument for measuring a quantum mechanical observable must have a macroscopic pointer, to be described classically as far as its macroscopic properties are concerned. But it is equally important that a measuring instrument have also a microscopic component which is sensitive to the microscopic information that in the measurement process is transferred to it from the microscopic object. Actually, quantum mechanics presents a clear paradigm of the 'theory-ladenness of observation', which during the second half of the $20^{\text {th }}$ century caused the decline of logical positivist/empiricist influence. A growing awareness of the necessity to consider a physical theory not as universally valid, but only as valid on a certain domain of application, has stimulated the view that application of classical notions to microscopic processes may not be such a good idea in general. The information transfer between the microscopic object and the sensitive part of the measuring instrument is a microscopic process, well within the domain of application of quantum mechanics.

It should be noted that the ban on a quantum mechanical description of measurement, imposed by the correspondence principle, has been disregarded e.g. by von Neumann and by Heisenberg. However, the quantum mechanical treatments by von Neumann and Heisenberg probably had the intention to justify the idea of 'correspondence' by means of quantum mechanical considerations, rather than to be independent attempts at a quantum mechanical account of measurement. As will be reviewed in section [10, a genuine application of quantum mechanics to quantum 
mechanical measurement is indispensable for an assessment of the role played by measurement in quantum mechanics. In particular, it follows that a generalization of the mathematical formalism is necessary in order to be able to describe even experiments that -like the two-slit experiment- were crucial in developing the idea of 'complementarity'. Also, von Neumann's projection postulate turns out to be obsolete as a measurement principle securing a well-defined value of an observable as a result of a measurement. It seems that the urge to interpret such a result as a property of the microscopic object is a consequence of the classical thinking involved in the correspondence principle.

The correspondence principle is consistent with the idea that a quantum mechanical observable does not have a value prior to its measurement. The contextual meaning of a quantum mechanical observable is justified by the essential role played by the measurement arrangement in its definition (cf. section 2.2). It seems that theorems like the Kochen-Specker and Bell theorems corroborate this idea. Therefore, in our attempt to develop a neo-Copenhagen interpretation of the quantum mechanical formalism this feature will be maintained: quantum mechanical observables are associated with the measurement arrangements set up to realize their measurement. Actually, since this feature is perhaps the prime characteristic of the Copenhagen interpretation, this is a reason to refer to the new interpretation as a neo-Copenhagen one, even though the latter will differ from it in many respects. In particular, I will take seriously the empiricist connotation of the correspondence principle mentioned above, by associating quantum mechanical observables with properties of the measuring instrument rather than with (contextual) properties of the microscopic object. This makes the essential role of the measurement arrangement in interpreting the quantum mechanical formalism even stronger. By doing so it will be seen in section [10 that the idea of 'complementarity' as a consequence of mutual disturbance in a joint measurement of incompatible observables need not be proposed as a separate principle, but that it straightforwardly follows from the mathematical formalism of quantum mechanics.

\section{$7 \quad$ EPR and correspondence}

There is an important lesson to be learnt from Bohr's [10] application of the correspondence principle in his answer to the EPR challenge. Actually, this application does not seem to be in complete agreement with this principle as given above. By

Einstein it was assumed that in the state (3) observables $A_{1}^{(s)}$ and $B_{2}^{(s)}$ are strictly correlated so as to allow inference of the value of one observable from the result 
obtained by measuring the other one. It is unfortunate that Bohr did not challenge this assumption on the basis of a strict application of the strong form of his correspondence principle. On the basis of this principle Bohr could have rejected the possibility of defining the correlation of observables $A_{1}^{(s)}$ and $B_{2}^{(s)}$ outside the measurement context for measuring it. A strict application of the correspondence principle (strong form) would have implied the requirement that measurements be performed on both particles, thus making obsolete the EPR idea of applying the notion of an 'element of physical reality', and, consequently, blocking the whole EPR reasoning. By taking into account that a definition of the correlation would imply that each of the particles is interacting with its own measuring instrument, Bohr could have maintained his contextualistic-realist interpretation in the interactionist sense originally intended, without any need to recede to a relational point of view (cf. section 5). Moreover, there would not have been any reason to resort to nonlocality in order to implement the contextuality going with the "nonlocal" measurement arrangement, since each particle's context can be locally determined by the measuring instrument the particle is interacting with.

However, Bohr did not challenge EPR in this way. Evidently, he did not recognize the correlation of observables $A_{1}^{(s)}$ and $B_{2}^{(s)}$ as an ordinary observable, to be measured by means of coincidence measurements like the EPR-Bell ones performed e.g. by Aspect et al. [42, 43]. Instead, he followed EPR in taking for granted existence of the correlation of the observables already if only one of the observables is measured. Consequently, in order to apply his correspondence principle Bohr had to refer to the context furnished for particle 2 by the measurement arrangement of particle 1 , thus starting the nonlocality enigma. On a consistent application of the correspondence principle we would not have had any reason to infer any nonlocality if measurements of the EPR-Bell type are considered rather than experiments of the EPR type.

Of course, this does not imply that such an inference of nonlocality could not be drawn in a different way from the EPR experiment as it was actually proposed. As already remarked in section [5, it is not possible to accept EPR's proposal to solve all problems by means of an ensemble interpretation of the state vector. In order to analyze this, it is necessary, however, to discuss another confusion inherent in the Copenhagen interpretation, viz. the confusion of the notions of 'preparation' and 'measurement', which also Bohr has seemingly fallen prey to in analyzing the EPR proposal, by accepting it as a measurement of an observable of particle 2 rather than as a preparation applied to this particle. 


\section{Preparation and measurement}

Since Bohr was ready to apply his correspondence principle (strong form) to the EPR experiment, he evidently accepted it as a measurement of a property of particle 2 , in the way intended by EPR. It is questionable, however, whether this is justified. Admittedly, on the basis of the existence of the strict correlation between observables $A_{1}^{(s)}$ and $B_{2}^{(s)}$, as suggested by (져), the measurement result of observable $B_{2}^{(s)}$, to be obtained if it were actually carried out in coincidence with the measurement of $A_{1}^{(s)}$, could be inferred already from the result of the measurement of the particle 1 observable. However, this inference is possible only if the correlation is assumed to exist independently of its measurement. Only on this basis can the measurement of particle 1 be interpreted as a measurement of the correlated property of particle 2. To the extent the strong form of the correspondence principle is an essential ingredient of the Copenhagen interpretation, this entails an internal inconsistency of this interpretation if it accepts the EPR experiment as a measurement of particle 2 .

In order to remove this inconsistency, it is necessary to duly distinguish between the EPR experiment and EPR-Bell experiments like the ones performed by Aspect et al., in the sense that in the former there is no measurement carried out on particle 2. Instead, by the EPR procedure this latter particle (better: the corresponding ensemble) is prepared in a certain state, conditional on a measurement result yielded by the measurement of particle 1 . Not distinguishing between the processes of 'measurement' and 'conditional preparation' has caused quite a bit of confusion. For instance, in an EPR-Bell experiment it would be possible to measure in coincidence with $A_{1}^{(s)}$ an observable $B_{2}^{\prime}$ of particle 2 , incompatible with the special observable

$B_{2}^{(s)}$. If the measurement of $A_{1}^{(s)}$ were still to be interpreted as a measurement of $B_{2}^{(s)}$, this would imply a simultaneous measurement of two incompatible observables of particle 2, which is in disagreement with another assumption of the Copenhagen interpretation, viz. the complementarity principle. As this latter principle is a cornerstone of the Copenhagen interpretation which is to survive (cf. section 10), it seems wise to prevent such a disagreement by duly distinguishing EPR experiments from EPR-Bell ones.

Unfortunately, the confusion of the notions of 'preparation' and 'measurement', observed here, is a general feature of the Copenhagen interpretation, induced by the impossibility of attributing an objective value of a quantum mechanical observable to the microscopic object, possessed prior to measurement. As a consequence, a measurement cannot reveal a pre-existing value of an observable, as is generally thought to be the case in classical mechanics. As a second best solution to this 
problem it was assumed that, at least, the value of the observable may be attributed to the object immediately after the measurement. This, actually, is the origin of von Neumann's projection postulate, intending the measurement to prepare the object in an eigenstate of the measured observable. Application of this postulate to the EPR experiment, considered as a measurement of observable $B_{2}^{(s)}$, has been responsible for the idea that the particle 2 state is projected onto one of the eigenstates $\left|\beta_{2 i}^{(s)}\right\rangle$. This idea has already been criticized by Margenau [44] on the basis that von Neumann's projection postulate is necessary only in an individual-particle interpretation of the wave function, and would be meaningless in an ensemble interpretation because a measurement on an individual particle cannot change the state of a whole ensemble.

Notwithstanding Margenau's criticism, the idea of a quantum mechanical measurement as preparing the microscopic object in one of a number of macroscopically distinguishable states, thus allowing to obtain a measurement result by ascertaining 'in which of these states the object finally is' (like, for instance, the Stern-Gerlach experiment), has become more or less paradigmatic of the Copenhagen interpretation. For instance, in his axiomatization of quantum mechanics, Jauch (45], section 11-3) considers a measurement as a filter, leaving the object in one of the eigenstates of the measured observable (so-called measurements of the first kind). By now it is well known, however, that the large majority of measurement procedures employed in actual practice does not satisfy this model. In general, it is not the microscopic object itself, but rather the pointer of a measuring instrument that is brought into one of a set of macroscopically distinguishable states. The microscopic object may even be annihilated in the measurement process (like, for instance, photons detected by an ideal photon counter). Conditional on the final pointer state the object is left behind in some state determined by the interaction of object and measuring instrument, in general differing from an eigenvector of the measured observable. For this reason it is hardly appropriate to consider von Neumann's projection postulate as a measurement principle, generally valid in quantum mechanical measurement.

However, this reasoning does not apply to EPR, because this experiment is not a measurement of particle 2. It is precisely due to this fact that von Neumann's projection postulate can be applied to EPR. Indeed, this postulate is a valid preparation principle, routinely applied in the laboratory for preparing microscopic objects in well-defined quantum mechanical states. Thus, if in the two-particle state (3) a measurement of an arbitrary observable $F_{2}$ of particle 2 is carried out, conditional on measurement result $a_{1 i}$ of the jointly measured observable $A_{1}^{(s)}$, then the conditional probability $p\left(f_{2 j} \mid a_{1 i}\right)$ of measurement result $f_{2 j}$ of $F_{2}$ can be derived from the joint probability

$$
p\left(a_{1 i}, f_{2 j}\right)=\left|\left\langle\psi_{12} \mid \alpha_{1 i}^{(s)} \phi_{2 j}\right\rangle\right|^{2}
$$


$\left(\left|\phi_{2 j}\right\rangle\right.$ the eigenvector of $F_{2}$ corresponding to eigenvalue $f_{2 j}$ ), according to

$$
p\left(f_{2 j} \mid a_{1 i}\right)=\frac{p\left(a_{1 i}, f_{2 j}\right)}{p\left(a_{1 i}\right)} .
$$

Putting

$$
p\left(f_{2 j} \mid a_{1 i}\right)=\left\langle\phi_{2 j}\left|\rho_{2 i}\right| \phi_{2 j}\right\rangle,
$$

it is straightforwardly proven that the density operator $\rho_{2 i}$ of the particle 2 state satisfying (77) is given by $\left|\beta_{2 i}^{(s)}\right\rangle\left\langle\beta_{2 i}^{(s)}\right|$. Hence, the subensemble of particles 2 prepared by selecting these particles on the basis of measurement result $a_{1 i}$ of observable $A_{1}^{(s)}$ of particle 1 from an ensemble of particle pairs prepared in state (3), is described by the state vector $\left|\beta_{2 i}^{(s)}\right\rangle$ postulated by von Neumann's projection postulate. Evidently, as a principle of conditional preparation this postulate has a certain legitimacy. It should be stressed that this legitimacy derives from the fact that the final state of the preparation process of particle 2 is not influenced by any interaction with a measuring instrument.

Measurements of the EPR type are sometimes called 'predictive measurements' (e.g. Kemble (46]) to distinguish them from 'determinative measurements' yielding only information about probabilities of the initial state, without telling anything about the post-measurement state of the microscopic object. In the former it is precisely this latter state that is the important issue, in the sense that we have here a method to prepare, conditional on the measurement result, the microscopic object in a well-defined state (like $\left|\beta_{2 i}^{(s)}\right\rangle$ in the EPR procedure). Here the preparation is the crucial issue: in the post-measurement state a (subsequent) measurement of an arbitrary observable $F_{2}$ can be performed. The EPR experiment is a (conditional) preparation of particle 2. Its interpretation as a measurement of an observable of this latter particle has caused much confusion. In particular, it seduced Bohr to apply his correspondence principle (strong form), even though it is a measurement principle rather than a preparation principle. Evidently, Bohr did not consider the observable $A_{1}^{(s)} \otimes B_{2}^{(s)}$, corresponding to a measurement of the correlation of the observables of particles 1 and 2, as an ordinary quantum mechanical observable, to be welldefined only within the context of the experimental arrangement for measuring it. By interpreting the conditional preparation of particle 2 as a measurement he went even so far as to assume that the correlation between the particles is determined by the measurement arrangement for only one of the particles (as well as, of course, by the preparing apparatus). The nonlocal contextuality ensuing from this must be seen as a consequence of an inconsistent application of the correspondence principle (strong form). 


\section{Conditional preparation versus contextual state}

The fundamental confusion of preparation and measurement, involved in the Copenhagen interpretation, can be highlighted in yet another way. For this purpose let us define for a system, prepared with state $\rho$, and on which a measurement is performed of standard observable $A=\sum_{m} a_{m} P_{m}$, the contextual state

$$
\rho_{A}=\sum_{m} P_{m} \rho P_{m}
$$

It is usual to consider this state as the final state of a von Neumann-Lüders measurement procedure, for which, in agreement with Heisenberg's ideas discussed above, the equality $\operatorname{Tr} \rho_{A} P_{m}=\operatorname{Tr} \rho P_{m}$ allows to infer the probabilities of the initial state from those measured in the final one. However, in view of the inapplicability of the notion of 'measurement of the first kind', this interpretation does not seem to be very useful. Bohr's correspondence principle (strong form) offers a way to look upon the state $\rho_{A}(8)$ in a different way, viz. as an alternative description of the initial state of an object, prepared according to $\rho$, as soon as it is within the context of the measurement arrangement for measuring observable $A$. Note that, due to Bohr's instrumentalist conception of quantum mechanical state vectors, presumably this interpretation is far from Bohrian. But it would at least yield another ontological implementation of Bohr's idea that observable $A$ is well-defined only within the context of a measurement of this observable, consistent with Bohr's correspondence view of observables discussed in section 6 . It would allow within quantum mechanics to distinguish between the state of an object as it is prepared prior to its interaction with a measuring instrument, and the state probed by this measuring instrument, possibly already influenced by that instrument. This is quite analogous to the way a classical rigid body model of a billiard ball accounts for certain observations, thus accounting for rigidity as a property of a billiard ball only to be attributed within a particular experimental context (cf. section 2.2, see section 12 for another analogy).

The contextual state should be distinguished from the conditionally prepared states of a measurement procedure. The distinction between a contextual state and a conditionally prepared state is particularly telling in the EPR experiment. As demonstrated in section 8 , in a measurement of the particle 1 observable $A_{1}^{(s)}$ in the two-particle state (3), particle 2 is conditionally prepared in the state $\left|\beta_{2 i}^{(s)}\right\rangle$. In the Copenhagen interpretation this is often interpreted in the sense that the corre-

sponding value of observable $B_{2}^{(s)}$ has got a definite value due to the measurement on particle 1. In Bohr's correspondence view of the EPR experiment it is even assumed that this value must be well-defined as soon as particle 1 is interacting with its measuring instrument, thus starting the nonlocality enigma. 
Now the question can be asked: does the state vector $\left|\beta_{2 i}^{(s)}\right\rangle$ "really" describe the reality of particle 2 as it is in the context of a measurement of an observable of particle 1? The problem is that we cannot tell. In order to probe this reality we have to make a measurement on particle 2 of some observable $F_{2}=\sum_{j} f_{2 j}\left|\phi_{2 j}\right\rangle\left\langle\phi_{2 j}\right|$. In the context of this measurement the contextual state is given by

$$
\left|\beta_{2 i}^{(s)}\right\rangle\left\langle\left.\beta_{2 i}^{(s)}\right|_{F_{2}}=\sum_{j}\left|\left\langle\beta_{2 i}^{(s)} \mid \phi_{2 j}\right\rangle\right|^{2} \mid \phi_{2 j}\right\rangle\left\langle\phi_{2 j}\right|
$$

This state is very different from $\left|\beta_{2 i}^{(s)}\right\rangle\left\langle\beta_{2 i}^{(s)}\right|$. Since these states yield the same measurement results for $F_{2}$, it is impossible to tell which is the "real" one. Whether before the measurement the state "really" was $\left|\beta_{2 i}^{(s)}\right\rangle\left\langle\beta_{2 i}^{(s)}\right|$ is a matter of interpretation. This will be discussed more extensively in section 11, where a different interpretation will be proposed.

More generally, for the two-particle system involved in an EPR-Bell measurement in which an arbitrary correlation observable $A_{1} \otimes B_{2}=\sum_{i} a_{1 i}\left|\alpha_{1 i}\right\rangle\left\langle\alpha_{1 i}\right| \otimes$ $\sum_{j} b_{2 j}\left|\beta_{2 j}\right\rangle\left\langle\beta_{2 j}\right|$ is measured in an arbitrary state $\left|\psi_{12}\right\rangle$, the contextual state (8) is given by

$$
\left|\psi_{12}\right\rangle\left\langle\left.\psi_{12}\right|_{A_{1} \otimes B_{2}}=\sum_{i j}\left|\left\langle\psi_{12} \mid \alpha_{1 i} \beta_{2 j}\right\rangle\right|^{2} \mid \alpha_{1 i} \beta_{2 j}\right\rangle\left\langle\alpha_{1 i} \beta_{2 j}\right| .
$$

From this expression the contextual states of the two particles separately can be derived by partial tracing as the contextual states to be found for each particle, independently of which measurement is carried out on the other particle. This illustrates the possibility of local contexts referred to in section 6. Contrary to what is often supposed, the conditionally prepared state need not play any prominent role in describing the reality of particle 2 if a measurement is carried out on this particle, because in that case this reality is locally (co-)determined by the measurement arrangement that is actually present at the location of this particle.

\section{Generalized observables, and complementar- ity}

As already noted in section 6, the Copenhagen confusion of the notions of 'preparation' and 'measurement' has had an equally confusing effect with respect to the issue of 'complementarity'. By Heisenberg 'mutual disturbance in a joint measurement of incompatible observables' was taken in a preparative sense, in which 'disturbance' is referring to the preparation of the final state of the object. There is another 
possibility, however, to the effect that 'disturbance' may refer to the final state of the measuring instrument, in the sense that the probability distribution of the final pointer positions may depend on the details of the measurement procedure, and, hence, may change if the measurement arrangement is changed so as to also yield information on an observable incompatible with the measured one. Then 'measurement disturbance' may refer to a deviation of such a probability distribution from the "ideal" one obtained in a measurement procedure reproducing the probability distribution predicted by the standard formalism of quantum mechanics. This will be referred to as 'mutual disturbance in a determinative sense'. Due to a restriction of the attention to measurements of the Heisenberg type in which measurement results are associated with final states of the microscopic object, the distinction between the preparative and determinative types of disturbance has remained largely unnoticed.

This subject has been more fully discussed elsewhere [47, 12, and will be reviewed here only briefly, even though it is of primary importance for understanding the ways in which the Copenhagen interpretation has been confused. It is important to note here that a major cause of this confusion was the fact that at the time Bohr and Heisenberg developed the complementarity principle the quantum mechanical formalism had not been fully developed. Conclusions with respect to uncertainties were often drawn from the classical reasoning advocated by the correspondence principle. As far as measurement was described quantum mechanically at all, this description seems to have served mainly to justify the idea of 'correspondence' (in particular, by means of von Neumann projection). Only after the quantum mechanical character of measurement in the atomic domain has been taken seriously, it has become possible to straighten out the above-mentioned confusion involved in the notion of 'complementarity'. It turns out that complementarity in the sense of 'mutual disturbance in a joint measurement of incompatible observables' need not be seen as a consequence of limitations of the classical reasoning involved in the correspondence principle, but that, if 'mutual disturbance' is taken in the determinative sense defined above, it can be derived from the mathematical formalism of quantum mechanics.

It had to be realized first, however, that the standard representation of a quantum mechanical observable as a Hermitian operator is too restricted a concept to encompass all possible measurements in the atomic domain (in particular, joint measurement of incompatible observables). This follows straightforwardly from a quantum mechanical treatment of the interaction of a microscopic object and a measuring instrument, by applying the usual measurement postulates to the mea- 
suring instrument. We find the quantum mechanical probabilities according to

$$
p_{m}=\operatorname{Tr}_{o} \rho M_{m}, M_{m}=\operatorname{Tr}_{a} \rho_{a} U^{\dagger} E_{m}^{(a)} U
$$

in which $\rho_{a}$ is the initial state of the measuring instrument, $U$ is the unitary evolution operator of the interaction process, and $E_{m}^{(a)}$ are operators of the measuring instrument $a$ determining the detection probabilities $p_{m}$ of the measurement as their final state expectation values. Compared to the standard formalism of textbook quantum mechanics the generalization consists of the fact that nothing requires the operators $M_{m}$ to be projection operators. In general, the quantum mechanical measurement results (detection probabilities) are represented according to (9) by the expectation values of a positive operator-valued measure (POVM) $\left\{M_{m}\right\}$ taken in the initial state $\rho$ of the microscopic object. It can be verified that the two-slit experiment -consideration of which has contributed in an essential way to the development of the notion of 'complementarity'- is not representable by a Hermitian operator (compare de Muynck [12, section 7.3). It is not surprising that much confusion has been generated by drawing general conclusions from a too restricted mathematical formalism, viz. the standard formalism restricting to the projection-valued measures (PVMs) corresponding to the spectral representations of Hermitian (better: selfadjoint) operators. In particular it has led to unjustifiedly interpreting inequality (41) as a consequence of mutual disturbance in a joint measurement of position and momentum, rather than as a representation of our restricted ability to prepare initial states (cf. Ballentine [40).

As demonstrated by Martens and de Muynck [4], the inapplicability of the Heisenberg inequality (44) to mutual disturbance in a joint measurement of incompatible observables does not imply that this latter feature of quantum mechanical measurement is not a perfectly real one. As a matter of fact, this feature has been demonstrated to exist in a number of 'thought experiments' widely discussed during the days of the inception of the idea of 'complementarity'. However, for its theoretical description it needs the generalization of the standard formalism referred to above. Whereas it is not at all clear how a joint measurement of incompatible standard observables could be described by the standard formalism, it is easy to define a joint measurement of incompatible observables (POVMs) $\left\{P_{m}\right\}$ and $\left\{Q_{n}\right\}$ by requiring that a bivariate POVM $\left\{R_{m n}\right\}$ exist, of which $\left\{P_{m}\right\}$ and $\left\{Q_{n}\right\}$ are marginals, such that the expectation value $\operatorname{Tr} \rho R_{m n}$ can be interpreted as the joint probability distribution of the latter observables. It is easy to find examples satisfying this requirement. In agreement with the standard formalism, this is impossible if $\left\{P_{m}\right\}$ and $\left\{Q_{n}\right\}$ are projection-valued measures of standard observables, represented by the corresponding Hermitian operators. 
More generally, it is possible to define a joint nonideal measurement of incompatible POVMs $\left\{P_{m}\right\}$ and $\left\{Q_{n}\right\}$ by requiring a bivariate POVM $\left\{R_{m n}^{\prime}\right\}$ to exist, such that

$$
\begin{aligned}
& \sum_{n} R_{m n}^{\prime}=\sum_{m^{\prime}} \lambda_{m m^{\prime}} P_{m^{\prime}}, \lambda_{m m^{\prime}} \geq 0, \sum_{m} \lambda_{m m^{\prime}}=1, \\
& \sum_{m} R_{m n}^{\prime}=\sum_{n^{\prime}} \mu_{n n^{\prime}} Q_{n^{\prime}}, \mu_{n n^{\prime}} \geq 0, \sum_{n} \mu_{n n^{\prime}}=1 .
\end{aligned}
$$

Here matrices $\left(\lambda_{m m^{\prime}}\right)$ and $\left(\mu_{n n^{\prime}}\right)$ are so-called nonideality matrices, defining the nonideality of the determination of probabilities of observables $\left\{P_{m}\right\}$ and $\left\{Q_{n}\right\}$, respectively, if POVM $\left\{R_{m n}^{\prime}\right\}$ is measured. It should be noted that $\left\{P_{m}\right\}$ and $\left\{Q_{n}\right\}$ may be PVMs here.

As measures of nonideality of the nonideality matrices $\left(\lambda_{m m^{\prime}}\right)$ and $\left(\mu_{n n^{\prime}}\right)$ it is useful to take the average row entropies (restricting to finite dimension $N$ )

$$
J_{(\lambda)}=-\frac{1}{N} \sum_{m m^{\prime}} \lambda_{m m^{\prime}} \ln \frac{\lambda_{m m^{\prime}}}{\sum_{m^{\prime \prime}} \lambda_{m m^{\prime \prime}}}
$$

(and analogously for $\left(\mu_{n n^{\prime}}\right)$ ). If $\left\{P_{m}\right\}$ and $\left\{Q_{n}\right\}$ are PVMs, it is possible [48] to derive for the nonideality measures $J_{(\lambda)}$ and $J_{(\mu)}$ the Martens inequality

$$
J_{(\lambda)}+J_{(\mu)} \geq-\ln \left\{\max _{m n} \operatorname{Tr} P_{m} Q_{n}\right\} .
$$

Note that the right hand side of inequality (12) vanishes if $\left\{P_{m}\right\}$ and $\left\{Q_{n}\right\}$ are compatible PVMs.

It should be stressed that the meaning of the Martens inequality is completely different from that of the Heisenberg inequality (4). In contrast to the latter inequality, the Martens inequality (12) is derived from the properties of the observables alone. It is a property of the POVM $\left\{R_{m n}^{\prime}\right\}$, which is independent of the initial state. The inequality is in an unambiguous way expressing the notion of 'mutual disturbance of measurement results in a joint measurement of incompatible observables', to the effect that the quantities $J_{(\lambda)}$ and $J_{(\mu)}$ can be seen as measures of the inaccuracies of the measurements of the observables $\left\{P_{m}\right\}$ and $\left\{Q_{n}\right\}$, respectively, caused by a mutual disturbance of these observables by the measurement process. In contrast to the Heisenberg inequality the Martens inequality is a perfect mathematical representation of the idea of 'complementarity' as illustrated by the 'thought experiments' (like the two-slit experiment and Heisenberg's $\gamma$-microscope). That this role was initially attributed to the Heisenberg inequality must be seen as an instance of 'jumping to conclusions', in the sense that the availability of the Heisenberg inequality seemed to corroborate so perfectly the idea of 'complementarity' as defined above that it clouded most physicists critical senses. Apart from a few critics like Einstein and Margenau, it had to wait for Ballentine's 1970 paper for an in-depth analysis of the 
inappropriateness of this attribution, by demonstrating that the Heisenberg inequality (4) is not a property of a 'simultaneous or joint measurement of incompatible observables' but rather a property of 'preparation' (i.e. of the initial state $|\Psi\rangle$ ).

That the confusion could last so long, has several causes. First of all, the Martens inequality (12) needs the generalized formalism for its derivation. This formalism was not available then. Moreover, the idea of 'completeness in a wider sense', applied to the standard formalism of quantum mechanics, may have been responsible for the acceptance of the Heisenberg inequality as the only candidate for representing so obvious a physical phenomenon as 'measurement inaccuracy due to mutual disturbance'. Only after the generalized formalism had been developed it was possible to break away from this paradigm by deriving from the generalized formalism the Martens inequality (12) as a better candidate for this purpose. In certain of the 'thought experiments' (for instance, the two-slit experiment with a moving screen) the measurement inaccuracy is a consequence of the uncertainties of position and momentum in the initial state of the screen, expressed by the Heisenberg uncertainty relation for the initial state of the screen. This makes it understandable that measurement inaccuracy was associated with the Heisenberg inequality. Another reason may be the confusion of preparation and measurement inherent in a restriction of the attention to measurements of the first kind, for which it is not implausible to interpret -as was done by Heisenberg- the standard deviations of the final state as measures of inaccuracy of measurement results to be attributed to the initial state.

Such confusions could arise because it was thought that no quantum mechanical analysis of the measurement process could be given (cf. section 61). It must be stressed that the developments leading to the generalized formalism of quantum mechanics could take place only on the basis of a rejection of this tenet of the Copenhagen interpretation. Only on this basis it was possible (de Muynck [47]) to realize that not one but two complementarity principles exist, one for preparation (expressed by the Heisenberg inequality), and one for the joint measurement of incompatible observables (expressed by the Martens inequality), both derivable from the (generalized) formalism.

\section{Realist versus empiricist interpretation of quan- tum mechanics}

An interpretation of a physical theory is a mapping from its mathematical formalism into the physical world. Roughly speaking there are two different possibilities for 
quantum mechanics, viz. a realist interpretation and an empiricist one. Here the possibility is ignored of an instrumentalist interpretation, to the effect that no mapping into reality is required at all but the theory is considered as 'an instrument for generating measurement results', because in the author's view this interpretation's omission of attributing a well-defined physical meaning to quantum mechanical concepts has been a source of confusion. This holds true in particular with respect to the notion of a 'quantum mechanical measurement result' which, due to instrumentalist vagueness, could be taken at will either as a property of the microscopic object, or as a property of the measuring instrument, or both.

In a realist interpretation of quantum mechanics the mapping is thought to be from the mathematical formalism into microscopic reality. The mathematical entities of the theory (state vector $|\Psi\rangle$, density operator $\rho$, standard observable $A$, and generalized observable $\left\{M_{m}\right\}$ ) are thought to represent properties of the microscopic object (realist interpretation of quantum mechanics).

A realist interpretation is very similar to the way classical mechanics is usually interpreted. It is the interpretation adopted in virtually all textbooks of quantum mechanics. Instruments used to prepare the microscopic object in an initial state, as well as measuring instruments, are not represented in the quantum mechanical description even if they are physically present.

As discussed already in section 2, we should distinguish objectivistic and contextualistic versions of a realist interpretation of quantum mechanics. In an objectivisticrealist interpretation the quantum mechanical description is thought to refer to objective reality, that is, a reality independent of any observer, including his measuring instruments. In a contextualistic-realist interpretation quantum mechanical concepts are thought to have a meaning only within a certain physical context (like the object's environment, or a measurement arrangement). Presumably as a consequence of an ill-understood classical paradigm, textbook presentations of quantum mechanics are generally cast into an objectivistic-realist terminology. However, as demonstrated by the billiard ball analogy discussed in section 2, not even classical theories do allow an objectivistic-realist interpretation.

In an empiricist interpretation of quantum mechanics the mapping of $|\Psi\rangle, \rho, A$, and $\left\{M_{m}\right\}$ is thought to be from the mathematical formalism into the macroscopic reality of instruments or procedures for preparing and measuring microscopic objects (empiricist interpretation of quantum mechanics).

Thus, in an empiricist interpretation a wave function or a density operator is thought to be a symbolic representation of a preparing apparatus (for instance, a cyclotron with specified knob settings) or a preparation procedure. A quantum mechanical 
observable (POVM or PVM) is thought to be a label of a measuring instrument (for instance, a photosensitive device for detecting photons) or a measurement procedure.

Even though the microscopic object is present, in an empiricist interpretation it is not thought to be represented by the quantum mechanical formalism. The quantum mechanical formalism is thought to describe 'just the phenomena', phenomena being situated in the macroscopic instruments for preparing and measuring the microscopic object. The empiricist interpretation as defined above is the weakest interpretation of quantum mechanics deploying a well-defined mapping of the mathematical formalism into reality. It comes closest to the way quantum mechanics is being used in the actual practice of experimental physics, if the physicist's tendency to devise (classical) pictures behind the phenomena is waived. The quantum mechanical formalism is thought not to provide any (causal) mechanisms explaining why a certain measurement result is obtained on a certain individual preparation. In particular, the formalism is thought to just describe EPR correlations, without expecting quantum mechanics to yield any causal explanation. Note that by itself this does not imply indeterminism as discussed in section 2.3. Quantum mechanics is thought to be neutral with respect to this latter issue, simply because the concept of a quantum mechanical observable does not apply to preparation procedures.

Notwithstanding similarities, an empiricist interpretation of quantum mechanics should be carefully distinguished from the empiricist view as fostered by the philosophical doctrine of logical positivism/empiricism, which considers metaphysical anything that is unobservable. In particular, an empiricist interpretation of quantum mechanics is perfectly consistent with a belief in a "real" existence of microscopic objects like atoms and electrons (reality behind the phenomena), even if these are not directly observed, nor described by this theory. Hence, an empiricist interpretation of quantum mechanics is not to be confused with the anti-realist philosophy referred to in section 2.1. According to an empiricist interpretation quantum mechanics does not describe the microscopic objects themselves, but just relations between preparing and measuring procedures, mediated by microscopic objects. It need not be assumed that correlations like those in EPR-Bell experiments do not have causes (as is done e.g. by van Fraassen [21]). Quantum mechanics just does not describe these causes. This can be compared to Newton's interpretation of his theory of gravitation, to the effect that this theory describes 'just the phenomena', and does not yield any (mechanical) explanation of the way the gravitational force is transmitted from the sun to a planet (about which Newton did not wish "to frame hypotheses"). The 'nonlocality' problem of quantum mechanics, started by the EPR challenge, is to be compared to the 'actio-in-distans' problem of the Newtonian theory of gravitation: both are consequences of a realist interpretation of the theory, 
asking from the theory explanations it is not able to give.

Hence, an empiricist interpretation of quantum mechanics is not at variance with metaphysical realism ("the theory that the objects of scientific enquiry exist and act, for the most part, quite independently of scientists and their activity" (Bashkar 49])). However, in order to describe the microscopic objects themselves new (subquantum) theories have to be developed, analogous to the field theories that nowadays are thought to be able to yield an explanation of Newton's 'action-at-adistance'. It should be stressed that, far from denying the possibility of subquantum (hidden-variables) theories, an empiricist interpretation of quantum mechanics leaves considerably more room for such theories than is allowed for by a realist interpretation, precisely because quantum mechanics is not thought to describe microscopic reality as such.

The empiricist interpretation should also be distinguished from the Copenhagen interpretation, which, although having an empiricist reputation, actually contains many realist elements. Nevertheless, the empiricist interpretation is indebted to the Copenhagen one by taking seriously the importance attributed to the role of the measuring instrument in assessing the meaning of the quantum mechanical formalism. But the empiricist interpretation differs from the Copenhagen one by not considering a quantum mechanical measurement result to be a property of the $m i$ croscopic object (either before, during, or after the measurement), but to correspond to a macroscopic event in the macroscopic part of a measuring instrument (pointer) that can be recorded in an unequivocal way either by direct observation or by means of registration by some memory device. Hence, the information transfer from the microscopic object to the measuring instrument must be taken into account. In agreement with the criticism of the correspondence principle given in section 6, the quantum mechanical formalism is applied to the interaction of object and measuring instrument.

One reason not to equate the professed empiricism of the Copenhagen interpretation with an empiricist interpretation of quantum mechanics as defined here, is that the discussion between Bohr and Einstein took place completely within the confines of a realist interpretation. For Einstein this was the natural interpretation, in which the reality described by a wave function is that of an ensemble, and an observable is thought to be an objective property of the microscopic object. Bohr departed from Einstein's interpretation of an observable only by replacing an objectivistically realist interpretation by a contextualistically realist one. Admittedly, Bohr's instrumentalist interpretation of the wave function, as well as his reference to "the possible types of predictions regarding the future behavior of the system" in his answer [10] 
to EPR sound vaguely empiricist. However, because Bohr, while leaning heavily on the strong form of the correspondence principle, restricted his considerations mainly to observables (physical quantities), this empiricism, if existing at all, did not carry much weight. In particular, his failure to notice the difference between EPR and EPR-Bell experiments demonstrates that for Bohr, like for Einstein, a quantum mechanical measurement result is a property of the microscopic object rather than a property of a macroscopic measuring instrument.

In many textbooks of quantum mechanics the way the state vector is dealt with can also hardly be distinguished from a realist one, even when adherence to the Copenhagen (orthodox) interpretation is acknowledged. The paradoxes stemming from this inclination towards realism have plagued the quantum mechanical literature over the years, and it has been realized many times that a more empiricist approach could solve most, if not all, problems (e.g. Wheeler [50]). On the other hand, it has been the Copenhagen reference to 'measurement' in the interpretation of quantum mechanics, which has been a bone of contention to those believing that quantum mechanics is applicable outside the context of a measurement. This is worded in a fairly dramatic way, for instance, by Popper (34, p. 2): "It seems to me that the attack on realism, though intellectually interesting and important, is quite unacceptable, especially after two world wars and the real suffering - avoidable suffering - that was wantonly produced by them; and that any argument against realism which is based on modern atomic theory - on quantum mechanics - ought to be silenced by the memory of the reality for the events of Hiroshima and Nagasaki." It seems to me, however, that judgments like Popper's one given above are based on an identification of empiricism and anti-realism, which is hardly defensible in general. It does not seem reasonable to treat a modest assessment of the meaning of a physical theory (as merely describing certain observed aspects of reality) as if this view would deny all reality behind the phenomena.

Perhaps Popper can be excused because, due to the idea of 'completeness of quantum mechanics in a wider sense' it could be thought that the Copenhagen interpretation endorses such an anti-realist view. However, as was seen in section 2.1. such a contention can hardly be attributed to the Copenhagen interpretation because it is rather 'completeness of quantum mechanics in a restricted sense' that is characteristic of this interpretation. Whether this latter concept is applicable to the violent events that took place in Hiroshima and Nagasaki, depends on the question of what is the domain of application of quantum mechanics. Application of quantum mechanics to macroscopic events may be possible to a certain extent, but we should be aware of the possibility that such events may transcend the domain of application of the theory (even though the theory may work well for a description of 
certain aspects of the microscopic processes that are involved). For a description of microscopic events quantum mechanics may play an analogous role to the one played by the theory of rigid bodies in describing solids: the theory may describe certain features of reality as they are under certain conditions defining the domain of application of the theory; under extreme conditions the theory may become inapplicable, and new theories will be needed to describe features of reality not covered by it. Unfortunately, in Popper's philosophy of science a theory's domain of application is largely absent.

Virtually all paradoxes that over the years have plagued quantum mechanics stem from a realist interpretation. Sometimes it is possible to alleviate a problem by relaxing from an individual-particle interpretation to an ensemble one, thus assuming, like in Schrödinger's cat paradox, that the wave function does not describe the reality of a single cat but of an ensemble of cats. However, the persistence of the so-called 'cross terms' in the density operator keeps provoking a necessity of appealing to 'observation' in order to account for their unobservability (e.g. Jauch [45], chapter 11). In contrast to Einstein's contention, an ensemble interpretation of the wave function does not solve all problems. The EPR problem, discussed in section [5, is a case in point here. An objectivistic-realist interpretation of conditional preparation of the particle 2 ensemble by a measurement on particle 1 keeps being problematic (although less acutely than in an individual-particle interpretation) because it attributes, for each member of the ensemble of particle pairs, to particle 2 sharp values of both incompatible observables $B_{2}^{(s)}$ and $B_{2}^{\prime}$. An empiricist interpretation offers an alternative to the nonlocality of Bohr's contextualistic realism by interpreting a transition from state vector $\left|\psi_{12}\right\rangle$ (3) to the state vector $\left|\beta_{2 i}^{(s)}\right\rangle$ not as a transition to a description of a subensemble of the ensemble of particles 2 , but as a transition to a new preparation procedure for particles 2, in which the latter particles are selected on the basis of the measurement results read off the measuring instrument for particle 1 . Since such a preparation procedure is manifestly causal the nonlocality problem does not arise.

It is important to note that an empiricist interpretation of EPR in this vein is not liable to the problem facing Einstein's solution on the basis of an objectivisticrealist ensemble interpretation, to the effect that according to the latter interpretation well-defined values of incompatible observables would be attributable to each particle of the ensemble, thus causing the problems of the Kochen-Specker and Bell type referred to in sections 2] and 6. In an empiricist interpretation in the EPR experiment particle 2 is not thought to acquire a well-defined value of $B_{2}^{(s)}$ as soon as a value of $A_{1}^{(s)}$ is ascertained. Such a value is obtained only if the observable is actually measured (as is the case in an EPR-Bell experiment). Hence, in a way 
an empiricist interpretation subscribes to Jordan's view that a quantum mechanical measurement result is created during a measurement. However, it is not created as a property of the microscopic object, but as a property of the measuring instrument (pointer position), which, for this reason, should be actually present. It should be noted that procedures like conditional preparation of particle 2 in the EPR experiment are not at all exceptional. They are quite common experimental procedures for preparing microscopic objects "in certain well-defined states" (for instance, the Compton-Simon experiment). Note, however, that in an empiricist interpretation the realist terminology, in which a state vector is thought to represent the result of a preparation, should be replaced to the effect that the wave function is rather representing the preparation itself. For describing states of the microscopic objects we have to take recourse to subquantum theories.

The neo-Copenhagen interpretation developed in the present paper endorses an empiricist interpretation of the quantum mechanical formalism. By thus weakening the interpretation paradoxes can be evaded. Far from "restrict(ing) quantum mechanics to be exclusively about piddling laboratory operations" this allows application of the theory to any preparation procedure representable by a quantum mechanical state vector or density operator: it is not necessary that the preparation be man-made. However, things are different with respect to observables (represented by Hermitian operators or POVMs). Quantum mechanics is intended in the first place to describe observations made by means of measuring instruments devised especially so as to create macroscopic phenomena, the relative frequencies of which can be registered conditionally on a certain preparation procedure. In the neo-Copenhagen interpretation quantum mechanics is thought just to describe relations between preparations and such measurement phenomena, which, however, are not to be interpreted in Bohr's realist sense but in the sense of an empiricist interpretation. Quantum mechanical measurement results refer to pointer positions of quantum mechanical measuring instruments. What is a quantum mechanical measuring instrument cannot be defined independently of the theory (i.e. quantum mechanics) itself. It is a matter of experience whether a certain experimental procedure is a valid measurement procedure within the domain of application of quantum mechanics. In particular, this domain has been appreciably extended as a consequence of the discovery that Hermitian operators do not exhaust all possibilities of representing quantum mechanical measurements. 


\section{Subquantum theories}

An interesting question, relevant to the neo-Copenhagen interpretation because it denies to quantum mechanics 'completeness in a wider sense', is a characterization of that theory's domain of application. As yet, we do not have any experimental clue helping us to answer this question, and necessary for knowing what kind of theories will be suitable for transcending quantum mechanics. Most attempts at devising subquantum theories had the intention to restore a classical, often deterministic, view (e.g. Bohm [51]). We do not have any reason, however, to think that at a submicroscopic level the world will be more similar to our macroscopic one than it is at the microscopic level.

Whereas the Copenhagen interpretation had the opportunity to neglect questions with respect to subquantum theories on the basis of a completeness claim of quantum mechanics (even though ill-understood), the neo-Copenhagen interpretation has to take these questions seriously. In particular, it has to cope with an alleged nonlocality, induced by derivations of the Bell inequalities from subquantum theories, even after it has been possible to banish the ghost of nonlocality at the quantum level by switching from a realist to an empiricist interpretation. However, as will be argued in the following, the nonlocality claim, based on violation of the Bell inequalities by certain EPR-Bell experiments, actually is a consequence of a completeness claim of the subquantum theory used, in the sense that it is assumed that this theory will describe all possible measurements, including quantum mechanical ones. It is not necessary to conclude that nonlocality is the cause of this violation if in the theory additional assumptions are made that are not satisfied in reality, and, therefore, make the theory inapplicable even if it is local. It is not even plausible that in case of quantum mechanical measurements nonlocality is the cause, because the possibility of violating the Bell inequalities by quantum mechanical measurements hinges on incompatibility of quantum mechanical observables, which, due to the (unchallenged) postulate of local commutativity, is a local affair. It is very well possible that the classical paradigm, referred to above, is such an additional assumption. In the following it is demonstrated how by getting rid of this paradigm also at the level of subquantum theories the ghost of nonlocality can be driven off.

Let us first briefly review derivations of the Bell inequalities from hidden vari-

ables theory. Allegedly ([52]) the most general one has been given by Clauser and Horne 53. It is based on the following representation of the quantum mechanical 
probability $p_{i}$ of measurement result $a_{i}$ of quantum mechanical observable $A$ :

$$
p_{i}=\int_{\Lambda} d \lambda \rho(\lambda) p_{A}\left(a_{i} \mid \lambda\right)
$$

Here $\lambda$ is a submicroscopic (hidden) variable, to be compared with the phase space point $(q, p)$ of classical mechanics, phase space being generalized to the hidden variables space $\Lambda$. The probability of $\lambda$ is given by $\rho(\lambda)$. The quantity $p_{A}\left(a_{i} \mid \lambda\right)$ is the conditional probability of measurement result $a_{i}$ for given $\lambda$. It actually is a representation of the detection process. If $p_{A}\left(a_{i} \mid \lambda\right)$ can have only values 0 and 1 the theory is called deterministic (this might implement the idea of 'faithful measurement', which does not have a meaning in an empiricist interpretation of quantum mechanics). It should be realized that this determinism refers only to the detection process; the free evolution in phase space, governing the time dependence of $\rho(\lambda)$, may be either deterministic or indeterministic. Since $p_{A}\left(a_{i} \mid \lambda\right)$ may depend on the measurement procedure used for measuring observable $A$, this theory can even account for contextuality of quantum mechanical observables. For EPR-Bell experiments (13) is generalized to

$$
p_{i j}=\int_{\Lambda} d \lambda \rho(\lambda) p_{A_{1} A_{2}}\left(a_{1 i}, a_{2 j} \mid \lambda\right), p_{A_{1} A_{2}}\left(a_{1 i}, a_{2 j} \mid \lambda\right)=p_{A_{1}}\left(a_{1 i} \mid \lambda\right) p_{A_{2}}\left(a_{2 j} \mid \lambda\right),
$$

in which the assumption of locality is implemented by a condition of conditional statistical independence of the bivariate conditional probabilities $p_{A_{1} A_{2}}\left(a_{1 i}, a_{2 j} \mid \lambda\right)$, the conditional probability of observable $A$ being independent of $B$ (and vice versa). A simple way to prove that the detection probabilities of the pairs of observables $\left(A_{1}, A_{2}\right),\left(A_{1}, B_{2}\right),\left(B_{1}, A_{2}\right)$, and $\left(B_{1}, B_{2}\right)$ satisfy the Bell inequalities (de Muynck et al. 54]) relies on the possibility to construct, on the basis of the validity of representation (14) for each of these measurements, the quadrivariate probability distribution

$$
p_{i j k \ell}=\int_{\Lambda} d \lambda \rho(\lambda) p_{A_{1}}\left(a_{1 i} \mid \lambda\right) p_{B_{1}}\left(b_{1 j} \mid \lambda\right) p_{A_{2}}\left(a_{2 k} \mid \lambda\right) p_{B_{2}}\left(b_{2 \ell} \mid \lambda\right) .
$$

The widely accepted idea that the quantum world must be nonlocal is based on the assumption that locality is the only presupposition in deriving the Bell inequalities from this theory, thus enabling to pinpoint nonlocality as the only possible cause of their violation in EPR-Bell measurements. Yet, there is still another assumption involved in the representations (13) and (14) of quantum mechanical detection probabilities. It is important to note that in these expressions by a conditional probability like $p_{A}\left(a_{i} \mid \lambda\right)$ quantum mechanical measurement results $a_{i}$ are 
conditioned on an instantaneous value of hidden variable $\lambda$. Is it reasonable to assume that a quantum mechanical measurement result $a_{i}$ is determined, even in a stochastic sense, by such an instantaneous value? If so, what is the precise instant of time at which the value of $\lambda$ should be taken in the conditional probabilities? Given the possibility that $\lambda$ is fluctuating very fast even compared to the characteristic transition times of quantum phenomena, it is probable that a quantum mechanical measurement does not probe an instantaneous value of $\lambda$ at all, but (a part of) a trajectory $\bar{\lambda}$, much in the same way a (thermodynamic) measurement of temperature or pressure does not probe an instantaneous value of $(q, p)$ in classical statistical thermodynamics. Subquantum theories of the types in which quantum mechanical measurement results are conditioned on $\lambda(\bar{\lambda})$ are referred to as quasi-objectivistic (non-quasi-objectivistic) subquantum theories in de Muynck [12].

The analogy between quantum mechanics and statistical thermodynamics has been drawn many times before (e.g. de Broglie [55], Bohm et al. [56, 57, Nelson [58, 59], Davidson [60], Dürr et al. 61]). If the analogy is sound, this implies that quantum mechanical measurements may not be fast enough to probe subquantum fluctuations. This means that for application to quantum mechanical measurements the conditional probabilities $p_{A}\left(a_{i} \mid \lambda\right)$ should probably be replaced by $p_{A}\left(a_{i} \mid \bar{\lambda}\right)$, and the expression (13) by a density functional like

$$
p_{i}=\int_{\bar{\Lambda}} d \bar{\lambda} \rho(\bar{\lambda}) p_{A}\left(a_{i} \mid \bar{\lambda}\right)
$$

in which $\bar{\Lambda}$ is a space of possible trajectories $\bar{\lambda}$, and $\rho(\bar{\lambda})$ is a probability distribution of these trajectories. The difference between $\lambda$ and $\bar{\lambda}$ marks the distinction between subquantum elements of physical reality and quantum mechanical ones, referred to in section 2.3 .

By itself a transition from (13) to (16) is not sufficient to block derivation of the Bell inequalities, since in (15), too, $\lambda$ could be replaced by $\bar{\lambda}$, thus allowing the construction of a quadrivariate probability distribution even if all conditional probabilities are conditioned on trajectories. In order to prevent such a construction it is useful to remember that, as far as quantum mechanical measurements probe reality, they probe a contextual reality (cf. section 9). In a subquantum theory describing such measurements this contextuality might be implemented by a contextuality of the trajectory $\bar{\lambda}$, which could be co-determined by the experimental arrangement in the same way as the canonical state $Z^{-1} e^{-H / k T}$ of classical statistical thermodynamics is co-determined by it (for instance, by the shape of the container of a gas). For this reason, in the context of a measurement of quantum mechanical observable $A$ in (16) the trajectory $\bar{\lambda}$ has to be replaced by a trajectory $\bar{\lambda}^{A}$ that is dependent 
on the measurement arrangement. Hence, instead of (16) we get

$$
p_{i}=\int_{\bar{\Lambda}^{A}} d \bar{\lambda}^{A} \rho\left(\bar{\lambda}^{A}\right) p_{A}\left(a_{i} \mid \bar{\lambda}^{A}\right)
$$

in which $\bar{\Lambda}^{A}$ is the space of trajectories allowed within the context of a measurement of observable $A$.

Contextuality implies that it is natural to assume that for incompatible observables $A$ and $B$ (characterized by mutually exclusive measurement arrangements) we in general have

$$
\bar{\lambda}^{A} \neq \bar{\lambda}^{B}
$$

Hence, even if in different experiments the object were prepared in the same hidden variable state $\lambda$, the trajectories are distinct if the measurement arrangements are mutually exclusive. For incompatible observables we also have

$$
\bar{\Lambda}^{A} \neq \bar{\Lambda}^{B} .
$$

Now the important point is that in the representations (17) of probability distributions of incompatible observables the conditional probabilities cannot be conditioned on the same states (trajectories). As a consequence, the construction, analogous to (15), of a quadrivariate probability distribution fails, and the Bell inequalities cannot be derived any more (even though these may be satisfied for measurements in the subquantum domain, fast enough to probe the instantaneous value of $\lambda$ ).

The assumption that quantum mechanical measurements do not probe the instantaneous value of $\lambda$, but only (contextual) trajectories $\bar{\lambda}^{A}$ is sufficient to break the unnatural connection widely supposed to exist between nonlocality and violation of the Bell inequalities. Derivation of the Bell inequalities for EPR-Bell experiments like those performed by Aspect et al. [42, 43] is impossible, then, because an object cannot be in the same state (trajectory) for all of the four different measurements involved. This explanation is in agreement with the fact that incompatibility is a necessary condition for the Bell inequalities to be violated. Since in an EPR-Bell experiment the trajectory of each of the particles of the particle pair can be thought to be locally co-determined by its own measurement arrangement, the solution proposed here for subquantum theory is essentially the same as the one proposed for quantum mechanics (cf. section 7 ). No nonlocal influences are necessary to violate the Bell inequalities.

It is interesting to consider the possibility that an individual preparation within the context of a measurement of observable $A$ might be describable by a trajectory $\bar{\lambda}^{\left(A, a_{i}\right)}$, yielding with certainty measurement result $a_{i}$ if the measurement is a faithful 
one. This would restore determinism of the measurement process, to the effect that a measurement result $a_{i}$ refers in a deterministic way to a subquantum state, be it a trajectory. A connection between quantum mechanics and the subquantum theory proposed here might be provided by the contextual state $\rho_{A}$ (8), which may be referring to the preparation of the subquantum states (trajectories) $\bar{\lambda}^{\left(A, a_{i}\right)}$ in an ensemble symbolically represented by the (statistical) state $\bar{\lambda}^{A}$, relative frequencies being given by (8). In general $\bar{\lambda}^{\left(A, a_{i}\right)} \neq \bar{\lambda}^{\left(B, b_{j}\right)}$ if $A$ and $B$ are incompatible.

Finally, it is interesting to note that the solution presented here corroborates a certain nonlocality of quantum mechanics (e.g. de Muynck 62]) quite different from the nonlocality involved in the usual explanation of violation of the Bell inequalities. This nonlocality has to do with the domain of application of a theory, delimiting the kind of objects described by it. Thus, an electron is not a point particle, but it is an extended object. However, within the domain of application of quantum mechanics it is to be considered as a (nonlocal/inseparable) whole, in the same way a billiard ball in the theory of rigid bodies, or a volume of gas in a thermodynamic equilibrium state is to be considered an inseparable whole. This does not imply that the objects are really nonlocal objects, in the interior of which nonlocal interactions would be responsible for inseparability, or for relative immobility of its parts. What it means is that within the domain of application of the relevant theory the objects can be considered as primitive entities. When experimentally leaving the domain of application of the theory the apparent nonlocality may disappear, analogously to the impossibility of staying within the domain of application of rigid body theory under experimental conditions capable of splitting a billiard ball.

\section{Summary}

In this paper an interpretation of the mathematical formalism of quantum mechanics is proposed, remedying the confusions and inconsistencies of the Copenhagen interpretation while maintaining the essential role attributed by the latter interpretation to the interaction of microscopic object and measuring instrument. For this latter reason it is proposed to refer to the new interpretation as a neo-Copenhagen one. It has particularly been inspired by the recent insight that, in order to encompass all possible measurements within the domain of quantum mechanics, it is necessary to generalize the mathematical representation of quantum mechanical observables to positive operator-valued measures, thus making obsolete the preferred position Hermitian operators have in the Copenhagen interpretation (as well as in most other interpretations). Thus, von Neumann's projection postulate is not applicable to 
generalized observables. Another reason to refer to the Copenhagen interpretation is that the notion of complementarity as a consequence of mutual disturbance in a joint measurement of incompatible observables remains one of the cornerstones of the new interpretation.

In the neo-Copenhagen interpretation the empiricist tendencies, to be observed in the Copenhagen interpretation but not implemented there in a consistent way, are taken seriously. By adopting an empiricist interpretation in which the mathematical formalism is taken to refer to macroscopic procedures of preparation and measurement rather than to the microscopic object, the paradoxes haunting quantum mechanics can be solved. In particular, the nonlocality problem, induced by the EPR experiment, does not exist any more. The contextual meaning of quantum mechanics, underlined by Bohr's analysis of the EPR proposal, is corroborated, but at the same time corrected by drawing a distinction between measurement and preparation, largely neglected in the Copenhagen interpretation. Bohr's instrumentalist interpretation of the wave function as well as Einstein's realist ensemble interpretation are shown to be wanting.

The discussion on the issue of the completeness of quantum mechanics is shown to be very confusing because two different notions of completeness are at stake. It is argued that an empiricist interpretation of quantum mechanics leaves room for subquantum theories. The concomitant nonlocality problem induced by the violation of the Bell inequalities is analyzed, and shown to be a consequence of a too restricted subquantum theory, unjustifiedly conditioning quantum mechanical measurement results on instantaneous values of the subquantum (hidden) variables. A comparison of the relation between quantum mechanics and such (quasi-objectivistic) subquantum theories with similar relations between theories describing physical phenomena at different levels of observation, demonstrates that presumably the assumption of quasi-objectivity cannot be maintained. Contrary to Bell's contention that nonlocality is the essential assumption allowing to derive the Bell inequalities, it is rather the assumption of quasi-objectivity (overlooked by Bell) that may be responsible.

\section{Acknowledgment}

The author thanks professor N.G. van Kampen and Peter Morgan for valuable remarks. 


\section{References}

[1] A. Einstein, Autobiographical notes, in Albert Einstein: Philosopher-Scientist, P.A. Schilpp ed., Cambridge University Press, London, Third edition, 1982, p. $1-94$.

[2] J.S. Bell, Against "measurement", in: Sixty Years of Uncertainty, A. Miller ed., Plenum, New York, 1990, p. 17.

[3] H. Everett, in: The many-world interpretation of quantum mechanics, B.S. DeWitt and N. Graham, eds., Princeton Univ. Press, 1973, p. 3.

[4] H. Folse, The philosophy of Niels Bohr, North Holland, Amsterdam, 1985.

[5] P.K. Feyerabend, Phil. of Science 35, 309 (1968).

[6] J. von Neumann, Mathematische Grundlagen der Quantenmechanik, Springer, Berlin, 1932; or, Mathematical foundations of quantum mechanics, Princeton Univ. Press, 1955.

[7] J.S. Bell, Rev. Mod. Phys. 38, 447 (1966).

[8] N. Bohr, in "Albert Einstein: Philosopher-Scientist", P.A. Schilpp, ed., The Library of Living Philosophers, 1949, p. 199.

[9] A. Einstein, B. Podolsky, and N. Rosen, Phys. Rev. 47, 777 (1935).

[10] N. Bohr, Phys. Rev. 48, 696 (1935).

[11] W. Kelvin, Philosophical Magazine 2, 1 (1901).

[12] Willem M. de Muynck, Foundations of quantum mechanics, an empiricist approach, Fundamental theories of physics, vol. 127, Kluwer Academic Publishers, Dordrecht, Boston, London, 2002.

[13] S. Kochen and E.P. Specker, J. Math. and Mech. 17, 59 (1967).

[14] N.D. Mermin, Phys. Rev. Lett. 65, 3373 (1990).

[15] N.D. Mermin, Physics Today, June 1990, 9.

[16] A. Peres, Journ. of Phys. 24A, L175 (1991).

[17] N.D. Mermin, Rev. Mod. Phys. 65, 803 (1993). 
[18] P. Jordan, Erkenntnis 4, 215 (1934).

[19] M. Redhead, Incompleteness, nonlocality, and realism, Clarendon Press, Oxford, 1987.

[20] P.K. Feyerabend, Zeitschr. f. Phys. 145, 421 (1956).

[21] B.C. van Fraassen, Quantum mechanics, an empiricists view, Clarendon Press, 1991.

[22] A. Einstein, Reply to criticisms, in Albert Einstein: Philosopher-Scientist, P.A. Schilpp ed., Cambridge University Press, London, Third edition, 1982, p. 665688.

[23] H. Primas, Chemistry, quantum mechanics, and reductionism, Springer-Verlag, Berlin, 1983.

[24] S.A. Werner and A.G. Klein, in Methods of Experimental Physics, K. Sköld and D.L. Price eds, Academic Press, Orlando, 1985, Vol. 23, Part A, p. 259.

[25] J. Summhammer, H. Rauch, and D. Tuppinger, Phys. Rev. A 36, 4447 (1987).

[26] A. Zeilinger, Phys. Lett. A 118, 1 (1986).

[27] D. Murdoch, Niels Bohr's Philosophy of Physics, Cambridge University Press, 1987.

[28] J.L. Park, Int. Journ. Theor. Phys. 8, 211 (1973).

[29] W. Band and J.L. Park, Found. of Phys. 6, 249 (1976).

[30] R. von Mises, Wahrscheinlichkeit, Statistik und Wahrheit, Springer, 1936.

[31] B. d'Espagnat, Conceptual Foundations of Quantum Mechanics, W.A. Benjamin, Inc., Reading, Mass., 1976.

[32] E. Schmidt, Math. Ann. 63, 433 (1906).

[33] C.A. Hooker, in: Paradigms and Paradoxes, R.G. Colodny, ed., Un of Pittsburgh Press, Pittsburgh, 1972, pp. 67-302.

[34] K.R. Popper, Quantum theory and the schism in physics, Rowman and Littlefield, Totowa, 1982.

[35] M. Jammer, The philosophy of quantum mechanics, Wiley, New York, 1974. 
[36] R. Guy and R. Deltete, Found. of Phys. 20, 943 (1990).

[37] A. Petersen, Quantum Physics and the Philosophical Tradition, M.I.T. Press, Cambridge, Mass., 1968.

[38] A. Messiah, Quantum mechanics, North-Holland Publ. Cy, Amsterdam, 1967.

[39] W. Heisenberg, The physical principles of quantum theory, Dover Publications, Inc., 1930.

[40] L.E. Ballentine, Rev. Mod. Phys. 42, 358 (1970).

[41] W. Heisenberg, Physics and Philosophy, Harper and Row, Publ., New York, 1962.

[42] A. Aspect, P. Grangier, and G. Roger, Phys. Rev. Lett 47, 460 (1981).

[43] A. Aspect, J. Dalibard, and G. Roger, Phys. Rev. Lett. 49, 1804 (1982).

[44] H. Margenau, Phys. Rev. 49, 240 (1936).

[45] J.M. Jauch, Foundations of Quantum Mechanics, Addison-Wesley Publ. Cy., Reading Mass., 1966.

[46] E.C. Kemble, The Fundamental Principles of Quantum Mechanics, McGrawHill Book Company, Inc., New York, 1937.

[47] W.M. de Muynck, Found. of Phys. 30, 205 (2000).

[48] H. Martens and W. de Muynck, Found. of Phys. 20, 255, 357 (1990).

[49] R. Bashkar, Scientific realism and human emancipation, Verso, London, New York, 1987.

[50] J.A. Wheeler, in: Problems in the Foundations of Physics, G. Toraldo di Francia, ed., North-Holland Publ. Cy., Amsterdam, New York, Oxford, 1979, p. 423.

[51] D. Bohm, Phys. Rev. 85, 166, 180 (1952).

[52] P.H. Eberhard, Nuov. Cim. 46B, 392 (1978).

[53] J.F. Clauser and M.A. Horne, Phys. Rev. D 10, 526 (1974).

[54] W.M. de Muynck, W. De Baere, and H. Martens, Found. of Phys. 24, 1589 (1994). 
[55] L. de Broglie, La thermodynamique de la particule isolée, Gauthier-Villars, 1964; L. de Broglie, Diverses question de mécanique et de thermodynamique classiques et relativistes, Springer-Verlag, 1995.

[56] D. Bohm, Phys. Rev. 89, 458 (1953).

[57] D. Bohm and J.-P. Vigier, Phys. Rev. 96, 208 (1954).

[58] E. Nelson, Dynamical theories of Brownian motion, Princeton University Press, 1967.

[59] E. Nelson, Quantum fluctuations, Princeton University Press, 1985.

[60] M.P. Davidson, Physica 96A, 465 (1979).

[61] D. Dürr, S. Goldstein, and N. Zanghí, Journ. Stat. Phys. 67, 843 (1992).

[62] W.M. de Muynck, Found. of Phys. 14, 199 (1984). 